CUAD. CONTAB. / BOGOTÁ, COLOMBIA, 16 (40): 73-109 / ENERO-ABRIL 2015 / 73

\title{
Estado de flujos de efectivo: aplicación de razonamientos algebraicos y de la NIC $7^{*}$
}

doi:10.11144/Javeriana.cc16-40.efea

\author{
Alicia Patricia Duque-Sánchez \\ Docente, Universidad del Atlántico; grupo de investiga- \\ ción $\mathrm{C}$ tres: Contabilidad, Contaduría y áreas Conexas. \\ Magíster en contabilidad, Universidad de Medellín. \\ Especialista en gerencia estratégica de costos y gestión, \\ Fundación Universidad Central, Bogotá. Especialista en \\ pedagogía para el desarrollo del aprendizaje autónomo, \\ Universidad Nacional Abierta y a Distancia, UNAD, \\ Barranquilla. \\ Correo electrónico: aliciaduque@mail.uniatlantico.edu.co
}

\footnotetext{
* Las pautas para elaborar el Estado de Flujos de Efectivo, EFE, tratados en este documento surgieron del trabajo de investigación Proceso de elaboración del Estado de Flujos de Efectivo con razonamientos lógicos, contables y matemáticos, elaborado en desarrollo de la maestría en contabilidad, Universidad de Medellín. El objetivo de este trabajo de investigación era coadyuvar al fortalecimiento del pensamiento crítico de los estudiantes del programa de contaduría pública, de la Universidad del Atlántico, mediante la propuesta de un proceso de elaboración del EFE. Un resumen y adaptación al método indirecto fue expuesto en la Universidad Nacional de Bogotá, el 27 de agosto de 2014, en el Segundo Congreso Global de Contabilidad y Finanzas Interges, con la ponencia La estructura del estado de flujos de efectivo obtenida a través de razonamientos contables y algebraicos.
} 
Resumen El presente documento explica cómo elaborar el Estado de Flujos de Efectivo, EFE, al aplicar razonamientos algebraicos, contables y la IAS 7. Las explicaciones específicas se basan en un caso expresado con símbolos de letras en lugar de las cifras, el cual corresponde a un ejemplo sencillo de una empresa comercial que incorpora los otros tres estados financieros, políticas contables e información adicional requerida. Lo expuesto constituye una demostración matemático-contable de la manera como se construye la estructura de presentación del Estado de Flujos de Efectivo por el método directo, a partir de la doble ecuación vertical del Estado de Situación Financiera.

El estudio riguroso de esta demostración posibilita su aplicación en casos prácticos de las organizaciones, por lo cual constituye un reto para fortalecer las habilidades de pensamiento de orden superior, con el aprendizaje a partir del texto.

Palabras clave Estado de flujos de efectivo; método directo; NIC 7; razonamiento algebraico.

\section{Códigos JEL M41, I21}

\section{Cash Flow Statements: Applying Algebraic and NIC 7 Reasonings}

\footnotetext{
Abstract This document explains how to make the Statement of Cash Flows, SCF, through the application of algebraic, accounting, and NIC 7 reasonings. The specific explanations are based in a case expressed with letter symbols instead of the figures, which corresponds to the simple case of a commercial company that includes the other three financial statements, accounting policies, and additional information required. The aforementioned constitutes a mathematic-accounting demonstration of how the structure of the presentation of the Cash Flow Statements through the direct method is built, using the double vertical equation of the Statement of Financial Status. A comprehensive study of this demonstration enables its application for practical cases in companies, and for this reason it constitutes a cha-
}

llenge for strengthening higher order thought abilities, by learning through text.

Keywords Statement of Cash Flows; IAS 7.

\section{Demonstração de Fluxos de Caixa: aplicação de razoamentos algébricos e da IAS 7}

Resumo o presente documento explica como elaborar a Demonstração de Fluxos de Caixa, DFC, ao aplicar razoamentos algébricos, contábeis e a IAS 7. As explicações específicas são baseadas num caso expressado com símbolos de letras em vez de cifras, que corresponde a um exemplo simples de uma empresa comercial que incorpora as outras três demonstrações financeiras, políticas contábeis e informação adicional requisitada. O exposto constitui uma demonstração matemático-contábil da maneira como é construída a estrutura de apresentação da Demonstração de Fluxos de Caixa pelo método direito, a partir da equação dupla vertical da Demonstração de Situação Financeira. O estudo rigoroso desta demonstração possibilita sua aplicação em casos práticos das organizações, pelo que constitui um desafio para fortalecer as habilidades de pensamento de ordem superior, com a aprendizagem a partir do texto.

Palavras-chave Demonstração de Fluxos de Caixa; IAS 7.

\section{Introducción}

El estado de flujos de efectivo (en adelante, EFE) da cuenta de la variación del efectivo y equivalente de efectivo, como resultado de la suma algebraica de los flujos de efectivo de las actividades de operación, inversión y financiación. Suministra información para determinar la capacidad de la entidad para generar efectivo y 
equivalentes de efectivo, y las necesidades que tiene para utilizarlos.

El presente documento tiene como objetivo explicar, con razonamientos algebraicos y contables, cómo se llega a la estructura del Estado de Flujos de Efectivo (EFE) por el método directo, partiendo de los saldos comparativos incluidos en el Estado de Situación Financiera (ESF), mediante la aplicación de un caso de una empresa comercial, utilizando símbolos de letras en lugar de las cifras.

Se utiliza el lenguaje algebraico aplicado a lo contable para lograr una generalización con restricción de dominio, dada esta por su limitación al caso específico planteado, con la intencionalidad de inducir al lector a fortalecer sus habilidades de pensamiento.

La generalización proporciona los elementos esenciales para que sea aplicado a otros casos en la práctica contable y, en general, permite lograr una comprensión más profunda sobre el EFE.

Este documento está dirigido a aquellos interesados en apropiarse de la manera como se construye la estructura de presentación del EFE por el método directo, y que cuentan con los siguientes conocimientos previos: lo básico del lenguaje contable, en aspectos como partida doble y naturaleza de las cuentas, y con aspectos esenciales del lenguaje algebraico, en cuanto a la utilización de símbolos, la comprensión del signo igual (=) como equivalencia, las propiedades de la igualdad y el uso de ecuaciones lineales.

Para un mayor provecho, se requiere que el lector acepte el reto de trascender la lectura de lo expuesto, para aplicar lo aprendido a otros escenarios diferentes a lo planteado en el caso específico. Esto se logra con la transferencia de la información, que consiste en aprender algo en una circunstancia y aplicarlo después en otra diferente (Sanz de Acedo-Lizarraga, 2014, p. 119), con lo cual fortalece sus habilidades de pensamiento de orden superior.

\section{Fundamentación e intencionalidades}

Este documento se fundamenta en tres aspectos básicos: primero, la importancia de la enseñanza de la elaboración del Estado de Flujos de Efectivo en el área de contabilidad financiera; segundo, las características del lenguaje algebraico que permite un desarrollo del razonamiento cuantitativo; y tercero, el aporte de esta lectura al fortalecimiento de las habilidades de pensamiento.

En la formación del profesional de Contaduría Pública, en el área de contabilidad financiera, se ubica la temática del Estado de Flujos de efectivo (su elaboración, análisis e interpretación), debido a que este es uno de los estados financieros que las empresas deben presentar, según NIC 1 (párrafo 10, literal d). Esta obligatoriedad normativa proviene de la importancia que constituye este estado financiero al proporcionar valiosa información para determinar la generación y uso de efectivo en las organizaciones.

La ausencia de abstracción en los procesos de enseñanza universitaria es una de las debilidades más serias de la educación contable (RincónSoto, Grajales-Londoño \& Zamorano-Ho, 2012, pie de página 33, p.124), por tanto, resulta pertinente que, como complemento a la formación 
específica de la elaboración del EFE, se desarrolle el razonamiento algebraico, que se caracteriza por generalizar y formalizar patrones (Godino \& Font, 2003, p. 774) y que implica el manejo de las abstracciones simbólicas del álgebra.

Comprender y aprender a partir del material escrito es una de las habilidades de pensamiento más importantes para el éxito (McNamara, 2004). El estudiante de educación superior debe aprender a partir de lo leído (Frade-Rubio, 2009, p. 88), teniendo en cuenta que para saber leer y aprender es indispensable saber pensar (Frade-Rubio, 2009, p. 81) y que trascender a la lectura, a lo aprendido es ejercitar la habilidad de transferencia, una de las habilidades de pensamiento de orden superior (García-Duque, 2006, p. 12; Muria-Vila \& Damián-Díaz, 2008, p. 143).

Belle Wallace en el prefacio del libro Competencias cognitivas en educación superior, de María Luisa Sanz de Acedo-Lizarraga (2014, pp. 9-10) indica que el mayor privilegio de los docentes universitarios es a su vez su mayor responsabilidad, que consiste en:

Particularmente la Universidad, apoyándose en su libertad para elaborar e implementar Planes de Estudio, debe estimular en los futuros profesionales esas capacidades para que puedan percibir la relevancia de sus aprendizajes, asociarlos a sus vidas, lograr una comprensión intuitiva de los demás, experimentar la alegría de la autorrealización, pensar por sí mismos, cuestionar la información, y la realidad social, justificar sus argumentos, tomar decisiones acertadas y solucionar problemas complejos.
Por lo anterior, las intencionalidades de este documento van encaminadas a pretender constituirse en una estrategia de fortalecimiento de habilidades intelectuales de orden superior que utiliza los contenidos declarativos y procedimentales propios del Estado de Flujos de Efectivo; tomando como contenido declarativo al "saber qué" (Díaz-Barriga Arceo \& Hernández-Rojas, 2010, p. 42) que va inserto en la NIC 7; mientras, el contenido procedimental, que es el "saber hacer" (Díaz-Barriga Arceo \& Hernández-Rojas, 2010, p. 44), constituye lo expuesto sobre la manera como se construye la estructura del EFE por el método directo.

\section{Aspectos generales del EFE}

\subsection{Normativa e importancia del EFE}

La contabilidad financiera está cambiando en el ámbito mundial, especialmente en los (de 2005 a 2015), atendiendo el llamado de la globalización de los mercados financieros. Esto se evidencia en que más de cien jurisdicciones se han acogido a los estándares internacionales de información financiera (Pacter, 2014, p. 6).

En Colombia, las Normas Internacionales de Contabilidad (NIC) y las Normas Internacionales de Información Financiera (NIIF) están incluidas en el Decreto 2784 de 2012 (específicamente en los anexos), el cual fue modificado parcialmente mediante el Decreto 3023 de 2013, con el que se actualiza la NIC 7 a la más reciente versión que aplica a partir de enero de 2014.

Estos decretos hacen parte de la reglamentación de la Ley 1314 de 2009, que regula los principios y normas de contabilidad en Colombia, y están dirigidos a los preparadores de la 
información financiera del Grupo 1, que corresponde a las grandes empresas (incluyendo emisores de valores y entidades de interés público), según lo establecido por el Direccionamiento estratégico, del Consejo Técnico de Contaduría Pública, CTCP (2012).

Según NIC 1, en el literal "d" del párrafo 10 establece que las empresas deben presentar el Estado de Flujos de Efectivo (EFE), junto con otros estados financieros, y luego, en los párrafos 47 y 111, indica que la NIC 7 es la normativa que establece los requisitos para la presentación y revelación de información sobre flujos de efectivo.

La NIC 7 fue emitida en 1977 por la IASC ${ }^{1}$ como Estado de Cambios en la Situación Financiera y, desde 1992 (con aplicación a partir de enero de 1994) se encarga del Estado de Flujos de Efectivo (EFE).

El EFE es importante para la actual economía, para la cual la liquidez es definitiva y fundamental, al permitir determinar las proyecciones de pagos y recaudos (Rincón-Soto, Grajales-Londoño \& Zamorano-Ho, 2012, p. 101). Resulta apropiado en el nuevo enfoque de la gestión del crédito y de la evaluación del riesgo crediticio, establecido por la comunidad financiera internacional, que empezó a enfocarse más en la liquidez de las organizaciones que en su solidez; y se empezó a leer la información hacia el futuro, más que hacia el pasado de la organización (Ostengo, 2009, p. 17).

\subsection{Componentes del EFE}

Los componentes básicos del EFE son:

1 IASC: International Accounting Standards Committee (en español: Comité de Estándares Internacionales de Contabilidad).
- Los flujos de efectivo de las actividades (operación, inversión y financiación), según párrafo 10 de la NIC 7.

- Variación del efectivo y equivalentes al efectivo, que es el resultado de la suma algebraica de los flujos de efectivo de las tres actividades.

Lo anterior se complementa con la adición del saldo inicial del efectivo y equivalentes, con lo que se obtiene el respectivo saldo final.

\subsection{Métodos de presentación del EFE} Según el párrafo 18 de la NIC 7, hay dos métodos para informar acerca de los flujos de efectivo de operaciones: directo e indirecto. La diferencia radica exclusivamente en la forma de presentación de los flujos de efectivo de la actividad de operación.

El método directo muestra los conceptos básicos de las entradas y salidas de efectivo de las partidas operativas; mientras que el método indirecto presenta la misma información en forma de conciliación, partiendo del resultado del ejercicio (Petti \& Longhi, 2007, p. 76). Las otras dos actividades (inversión y financiación) son iguales para los dos métodos de presentación del EFE.

El método indirecto parte del resultado del ejercicio, se le suman y restan los ajustes para dejarlo en términos del efectivo y se colocan las variaciones de las cuentas del activo corriente y del pasivo corriente, relativas a la operación del ente (excluyendo las cuentas de inversiones y de obligaciones financieras).

Aunque el párrafo 19 de la NIC 7 recomienda la presentación del EFE por el método directo argumentando que este método puede 
ser útil en la estimación de flujos de efectivo futuros; en la práctica, el método indirecto es el que predomina, al parecer "son pocas las empresas en Latinoamérica que utilizan dicho método por considerarse engorroso, complicado y difícil de preparar" (Estupiñán-Gaitán, 2009, p. xii). En Colombia, es muy utilizado el método indirecto, por ejemplo, la Superintendencia de Sociedades solicita información de los estados financieros básicos en formatos preestablecidos, en los que incluye el EFE, con la estructura de presentación del método indirecto.

\subsection{Presentación y revelación de la información en el EFE según NIC 7}

A continuación, se indican los aspectos básicos de la presentación y revelación de la información del EFE, de acuerdo a lo contemplado en la NIC 7.

\subsubsection{Saldo del efectivo y equivalentes de efectivo}

Con base en lo estipulado en el párrafo 6, se determina que el saldo del efectivo lo conforman los saldos de las cuentas de caja y bancos, disponibles sin ningún tipo de restricción; mientras que los equivalentes de efectivo son inversiones a corto plazo de gran liquidez, fácilmente convertibles en efectivo y sujetos a un riesgo poco significativo de cambios en su valor. A este respecto, añade en el párrafo 7, que para que una inversión sea equivalente de efectivo debe tener vencimiento próximo, por ejemplo, tres meses o menos desde la fecha de adquisición.

Con lo que define el párrafo 8, permite incluir como efectivo y equivalentes de efectivo los sobregiros bancarios que corresponden a la gestión del efectivo de la empresa. Es decir, cuando los sobregiros bancarios constituyen recursos de dineros disponibles, previamente convenidos y autorizados por la entidad bancaria.

Según el párrafo 45, las empresas deberán presentar una conciliación entre lo reportado como efectivo y equivalente al efectivo en el EFE y los rubros reportados en el ESF. Además, deberán revelar los criterios utilizados para determinar los saldos de efectivo y equivalentes de efectivo (párrafo 46).

\subsubsection{Información de flujos de efectivo en términos netos}

En la estructura de presentación del EFE por el método directo, de acuerdo a lo estipulado en el párrafo 12, en términos netos, se determinan los flujos de efectivo de la actividad de operación por los conceptos de cobros a clientes, pagos a los proveedores y a empleados; debido a que su rotación es muy alta, con vencimientos próximos y que en cuanto al total de cartera, generalmente corresponde un gran porcentaje con respecto al total del activo.

\subsubsection{Flujos de efectivo en moneda extranjera}

El párrafo 25 se refiere a que los flujos de efectivo que correspondan a transacciones en moneda extranjera se convertirán a la moneda funcional de la entidad, aplicando la tasa de cambio entre ambas monedas en la fecha en que se produjo cada flujo en cuestión.

Y luego, el párrafo 28 establece que no constituyen flujos de efectivo las pérdidas o ganancias no realizadas, producto de los ajustes por diferencias 
en cambio, y que tales ajustes se presentarán en la conciliación del inicio y final del efectivo y equivalentes del EFE, de manera que se indique este saldo sin y con ajuste por la diferencia en cambio.

\subsubsection{Intereses y dividendos}

En el párrafo 31 indica que los intereses y dividendos, recibidos y pagados, deben ser revelados por separado (sin importar que se hayan capitalizado, acorde con lo contemplado en el párrafo 32 de la NIC 7), e indica que ante la falta de consenso en las entidades no financieras para definir en dónde son clasificados los flujos de efectivo que se desprenden de estos conceptos (párrafo 33), permite varias alternativas de presentación. La que se escoja deberá ser definida de forma coherente.

\subsubsection{Intereses y dividendos recibidos}

Los flujos de efectivo por conceptos de intereses recibidos y por dividendos recibidos pueden ubicarse en las actividades de operación o de inversión (párrafo 33).

\subsubsection{Intereses pagados}

El flujo de efectivo por concepto de intereses pagados puede colocarse en las actividades de operación o de financiación (párrafo 33).

\subsubsection{Dividendos pagados}

El párrafo 34 estipula que los dividendos pagados pueden clasificarse como flujos de efectivo en las actividades financieras o de operación.

\subsubsection{Impuestos a las ganancias}

En el párrafo 35 establece que los pagos por concepto de impuesto a las ganancias deben mostrarse en forma separada, en las actividades de operación, a menos que se encuentren específicamente asociados con las actividades de inversión o de financiación.

\subsubsection{Transacciones no monetarias}

En los párrafos 43 y 44 se refiere expresamente a que las transacciones de inversión o financiación que no requieren el uso de efectivo o equivalentes al efectivo se excluyan del EFE, y que deben ser reveladas en cualquier otra parte de los estados financieros. Aunque no hace alusión a las partidas operacionales, se debe tener en cuenta que para la elaboración del EFE por el método directo, las variaciones de las cuentas del activo corriente y/o del pasivo corriente que no afectan el efectivo, por ejemplo, provisiones de cartera o de beneficios a empleados, deben ser excluidas del EFE.

\subsubsection{Otra información a revelar}

En el párrafo 48 establece que se deberá revelar cualquier importe significativo de saldos de efectivo y equivalentes al efectivo que no esté disponible para ser utilizado.

\subsection{Presentación del EFE por el método directo}

La presentación del EFE está conformada por el encabezado, el cuerpo del EFE, los saldos del efectivo y equivalentes de efectivo (inicial y final), como se aprecia en el ejemplo de la tabla 1. Se complementa con aspectos como el pie de firma, en el que se anotan los nombres y cargos de quienes se responsabilizan por el suministro de la información, su elaboración y revisión, según se requiera para darle validez legal. 
Empresa Ejemplo S.A.

Estado de flujos de efectivo

Desde el 1 de enero hasta el 31 de diciembre de

$$
2014
$$

Actividades de operación

\begin{tabular}{|c|c|}
\hline Recaudo de clientes & 400 \\
\hline Pago a proveedores & -60 \\
\hline Pago a empleados & -180 \\
\hline Recaudo de intereses & +50 \\
\hline Pago de intereses & -30 \\
\hline Pago de impuesto sobre las ganancias & -60 \\
\hline Otros pagos & -70 \\
\hline $\begin{array}{l}\text { Flujo neto de efectivo de las actividades de } \\
\text { operación }\end{array}$ & 50 \\
\hline \multicolumn{2}{|l|}{ Actividades de inversión } \\
\hline Inversiones redimidas & 10 \\
\hline Nueva constitución de CDT & -35 \\
\hline Recaudo por venta de equipo & +13 \\
\hline $\begin{array}{l}\text { Flujo neto de efectivo de las actividades de } \\
\text { inversión }\end{array}$ & -12 \\
\hline \multicolumn{2}{|l|}{ Actividades de financiación } \\
\hline Nuevas obligaciones financieras & 80 \\
\hline Pago de obligaciones financieras & -100 \\
\hline Pago de dividendos & -25 \\
\hline $\begin{array}{l}\text { Flujo neto de efectivo de las actividades de } \\
\text { financiación }\end{array}$ & -45 \\
\hline $\begin{array}{l}\text { Disminución del efectivo y equivalentes de } \\
\text { efectivo }\end{array}$ & -7 \\
\hline
\end{tabular}

efectivo

Saldo inicial del efectivo y equivalentes de efectivo

Saldo final del efectivo y equivalentes de efectivo

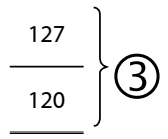

$1=$ Encabezado del EFE

2 = Cuerpo del EFE

3 = Saldos del efectivo y equivalentes de efectivo (inicial y final)

Tabla 1. Ejemplo de presentación del Estado de Flujos de Efectivo, EFE, por el método directo

Fuente: elaboración propia

A su vez, el cuerpo del EFE está compuesto por la ecuación del EFE, los títulos de cada ac- tividad y los respectivos totales. En la tabla 2 se muestra la ecuación ${ }^{2}$ del EFE correspondiente al ejemplo de presentación del EFE.

Hay que tener en cuenta que este documento trata indistintamente los conceptos de ecuación e igualdad, aunque en términos matemáticos la ecuación es una igualdad en la que hay una o más cantidades literales desconocidas.

$\begin{array}{lc}\text { Recaudo de clientes } & 400 \\ \text { Pago a proveedores } & -60 \\ \text { Pago a empleados } & -180 \\ \text { Recaudo de intereses } & +50 \\ \text { Pago de intereses } & -30 \\ \text { Pago de impuesto sobre las ganancias } & -60 \\ \text { Otros pagos } & -70 \\ \text { Inversiones redimidas } & +10 \\ \text { Nueva constitución de CDT } & -35 \\ \text { Recaudo por venta de equipo } & +13 \\ \text { Nuevas obligaciones financieras } & +80 \\ \text { Pago de obligaciones financieras } & -100 \\ \text { Pago de dividendos } & -25\end{array}$

Disminución del efectivo y equivalentes de efectivo

Tabla 2. Ejemplo de ecuación del Estado de Flujos de Efectivo, EFE, por el método directo

Fuente: elaboración propia

\section{Proceso de elaboración del EFE por el método directo con razonamientos algebraicos y contables}

El proceso para elaborar el EFE, que toma como hilo conductor la ecuación central, inicia

2 En el ejemplo de la tabla 2 no hay incógnitas. En términos matemáticos, se trata de una igualdad y no de una ecuación; pero este documento trata indistintamente esos dos términos. 
con las dos columnas ${ }^{3}$ contenidas en el Estado de Situación Financiera (ESF) que se transforman en la ecuación preliminar del EFE, luego en la ecuación del EFE por el método directo (ver ejemplo en la tabla 2) y por último, se completa la presentación del EFE.

El proceso de elaboración del EFE explicado, con sus fases, tareas y pasos expuestos, tiende a constituir un camino a seguir. Esto es recomendable al inicio, pero se sugiere que en la medida en que se apropie el procedimiento, cada lector le impregne su propio estilo, entonces, puede cambiar el rumbo para llegar a la misma meta, con base en sus conocimientos previos y en su manera de establecer las ecuaciones temporales y de transformar la ecuación central.

El proceso de elaboración del EFE por el método directo con razonamientos algebraicos y contables se enfoca en demostrar formalmente cómo se obtiene la ecuación del EFE, a partir de la información del Estado de Situación Financiera. Este tipo de demostración se caracteriza por establecer conexiones lógicas (Gómez-Marín, 2012, p. 224); utiliza el lenguaje algebraico y el lenguaje contable que, como todos los lenguajes artificiales o formales, son sistemas simbólicos abstractos construidos por el hombre con fines específicos de conocimiento (Gómez-Marín, 2012, p. 87); de manera que, en caso de que la normativa contable cambie con respecto a la presentación y revelación del EFE, estos cambios serían más fácilmente asimilados.

La demostración indica cómo se obtiene la ecuación del EFE por el método directo, partiendo de la doble ecuación del Estado de Si-

3 Las dos columnas corresponden a los saldos comparativos de dos años consecutivos. tuación Financiera. Esta es una demostración, porque justifica de manera rigurosa, mediante procesos formales deductivos, que se apoya en postulados matemáticos (García-Restrepo, 2007, p. 269), se complementa con planteamientos contables y se circunscribe a la normativa contable de la NIC 7.

\subsection{Información necesaria para elaborar el EFE}

La información necesaria para elaborar el EFE consta de:

- El Estado de Situación Financiera (ESF) comparativo al corte de los últimos dos períodos.

- El estado de resultados del último período.

- El estado de cambios en el patrimonio del último período.

- Las políticas contables referentes a la presentación y las revelaciones del EFE.

- La información adicional requerida para conformar los flujos de efectivo.

La información adicional requerida es aquella que complementa o confirma la información incluida en los otros tres estados financieros y en las políticas contables. Por lo tanto, permite determinar y/o corroborar ciertos flujos de efectivo, que de acuerdo a la NIC 7 se deben especificar ${ }^{4}$.

Esta información está disponible en las notas a los estados financieros, en los auxiliares de las cuentas contables, en los registros contables y en la información suministrada por

4 La NIC 7 establece que se deben revelar por separado los flujos de efectivo por concepto de los intereses recibidos y pagados, los dividendos recibidos y pagados, y los pagos por impuesto a las ganancias. 
áreas, como Tesorería y/o los encargados de la Gerencia Financiera de la empresa, que suministran información sobre algunos recaudos y pagos específicos que controlan, como venta de activos fijos, control de obligaciones financieras (adquisición de nuevas obligaciones y pagos realizados), control de inversiones (nuevas inversiones realizadas, compradas y las que se redimen o venden); cuyo número de operaciones es menor con respecto a las de la actividad principal que se dedica la empresa. Por esto, los flujos de efectivo que se refieren a la actividad principal de la empresa, por tener el mayor número de transacciones, se calculan de manera global, como recaudo por ventas, pago a proveedores y pago a empleados, entre otras.

\subsection{Símbolo en letras}

En el presente documento, las letras en mayúscula y en minúscula son utilizadas a manera de símbolos que reemplazan los valores, y se identifican con los conceptos o cuentas que atañen. Representan números en forma generalizada $^{5}$ (Casallas-Gómez \& Estrella, 2006, p. 130), que se restringen a los posibles valores del caso específico planteado y a aspectos propios del razonamiento contable. Entonces, en el lenguaje algebraico usado, no interesa llegar a conocer o reemplazar las letras por valores, porque mediante los símbolos en letras se realizan los análisis y operaciones matemáticas de acuerdo con las argumentaciones contables.

5 Esta es una opción, de las tres planteadas por Luz Marina Casallas-Gómez y Claudia Patricia Estrella (2006, p. 130), y las otras dos son: tomar "la letra como incógnita (en situaciones donde se requiere encontrar el valor de una de las variables cuando la otra toma un valor cualquiera)" y "la letra evaluada (cuando verifican la simbolización que encontraron)".
Para facilitar la ubicación de los valores que representan estos símbolos en letras, se han distribuido en tres listas de símbolos (ver tablas 3, 4 y 5). La tabla 3 indica los saldos del Estado de Situación Financiera (ESF), la 4 indica los valores que se usan en el Estado de Resultados y en el desglose de variaciones y, por último, la 5 representa los valores totales y subtotales incluidos en los cuatros estados financieros básicos.

\subsection{Equivalencia en la ecuación contable}

El concepto de equivalencia se aprecia desde la ecuación central 1, pues los saldos de las cuentas que conforman el activo, de naturaleza débito, se presentan con signo positivo ${ }^{6}$; y los saldos de las cuentas del pasivo y del patrimonio se presentan también con signo positivo, aunque son de naturaleza crédito, "contraria a la naturaleza de las cuentas del activo".

Esta noción de equivalencia entre los saldos débitos y créditos es esencial para el desarrollo de los razonamientos algebraicos. Se observa en la expresión contable que indica:

El total del activo = La sumatoria del total del pasivo y el total del patrimonio

\subsection{Subíndices en los símbolos de valores de los saldos, totales y subtotales del ESF}

Los subíndices 1 y 2 se utilizan en los símbolos que representan valores de los saldos, totales y subtotales ubicados en el ESF para diferenciar lo que corresponde de un año a otro; debido a que el

6 A excepción del saldo de depreciación acumulado, cuya naturaleza es crédito, porque tiene la cualidad de disminuir el valor de los activos fijos. 
ESF presenta la información comparativa de dos años sucesivos. Entonces, en el caso planteado, el subíndice 2 expresa los saldos, totales y subtotales que conciernen al año 2015 (el año más reciente), mientras que el subíndice 1 , le corresponde al año 2014 (año inmediatamente anterior).

\subsection{Ecuación central}

La ecuación central se presenta en forma vertical, es decir, tiene un extremo en la parte superior y otro en la parte inferior; separados por una doble línea gruesa, que representa el signo igual.

Resulta importante utilizar esta estructura en forma vertical, debido a que este es el esquema característico de la presentación del EFE, que se aprecia claramente en el ejemplo de la ecuación del EFE de la Tabla 2, pues la suma algebraica del extremo superior es igual a la variación del efectivo y equivalentes de efectivo, ubicada en el extremo inferior.

La ecuación central es aquella que va transformándose a lo largo del proceso de elaboración del EFE. Cada ecuación central va enumerándose a medida que va alcanzando los cambios establecidos por las diferentes tareas, ya sea por cambios en la estructura (de dos columnas pasa a tres, luego a una), por la reubicación de la secuencia de sus componentes (por medio del despeje de ecuaciones o agrupación según clasificación de la información) o por la sustitución de sus partes por sus equivalentes, mediante el uso de las ecuaciones temporales.

\subsection{Ecuaciones temporales}

Las ecuaciones temporales se expresan de manera horizontal y se incorporan a la ecuación central al reemplazar las variaciones de las cuentas por su equivalente.

Las ecuaciones temporales se utilizan para indicar la manera como se va reemplazando una información por otra, con el fin de ir depurando la información hasta obtener los flujos de efectivo. Primero, se obtienen los flujos de efectivo de las actividades de inversión y financiación y, por último, la conformación de flujos de efectivo de la actividad de operación, propios del método directo.

Las ecuaciones temporales se encuentran numeradas con un subíndice y se identifican con la abreviatura Et entre paréntesis.

\subsection{Fases y tareas del proceso para elaborar el EFE por el método directo}

El proceso para elaborar el EFE por el método directo que aplica razonamientos algebraicos, contables y NIC 7 está compuesto por cuatro fases; estas a su vez constan de un determinado número de tareas que a continuación se indican y que para su realización requieren pasos, que se detallan en el caso específico, para una apropiada explicación.

A continuación, se indican las cuatro fases con las tareas a realizar:

- Fase 1: conformación de la ecuación preliminar de la variación del efectivo y equivalentes

- Doble ecuación del ESF.

- Triple ecuación del ESF.

- Ecuación de las variaciones del ESF.

- Despeje de la variación del efectivo y equivalentes.

- Cambio de signos en ambos extremos. 
- Fase 2: depuración de la ecuación preliminar de la variación del efectivo y equivalentes

- Depuración de la variación del patrimonio (en dos partes: primero, sin incluir el detalle del resultado del ejercicio; y segundo, se hace el desglose de este).

- Depuración de las variaciones de las cuentas clasificadas como activos no corrientes.

- Depuración de las variaciones de las cuentas de inversión, clasificadas en el activo corriente.

- Depuración de las variaciones de las cuentas clasificadas como pasivos no corrientes.

- Depuración de las variaciones de las cuentas que contabilizan las obligaciones financieras, en el activo corriente.

- Fase 3: preparación de la ecuación del EFE por el método directo

- Identificación de los flujos de efectivo de las actividades de inversión y financiación, y de aquellas partidas para eliminar (por no afectar el efectivo y equivalentes). Las demás partidas se señalan como actividades de operación.

- Retiro de ítems identificados para eliminar y agrupación por las actividades del EFE (en el orden: operación, inversión y financiación).

- Identificación y asignación para conformar los flujos de efectivo de la actividad de operación, propios del método directo.
- Agrupación de ítems para conformar los flujos de efectivo de la actividad de operación.

- Determinación de las ecuaciones temporales que agrupan los flujos de efectivo de la actividad de operación.

- Revisión y ajuste de los flujos de efectivo de la actividad de operación.

- Conformación definitiva de los flujos de efectivo de la actividad de operación.

- Fase 4: presentación completa del EFE por el método directo

A la ecuación del EFE obtenida se le adicionan los aspectos complementarios necesarios para lograr la presentación completa del EFE por el método directo. Se adicionan los saldos del efectivo y equivalentes inicial y final, se coloca el título a cada actividad con sus respectivos subtotales, y se coloca el encabezado del EFE.

\section{Caso planteado para elaborar el EFE}

El caso planteado, mediante el cual se explica el proceso de elaboración del EFE por el método directo aplicando razonamientos algebraicos y contables, abarca el período comprendido desde el 1 de enero hasta el 31 de diciembre de 2015, y corresponde a la sociedad anónima denominada ADS, ubicada en el grupo 1 conforme al Decreto 2784 de 2012, que se dedica a la actividad de comercio al por mayor. 


\subsection{Listas de símbolos para representar los valores}

Las siguientes son las listas de símbolos que representan los valores usados a lo largo de la elaboración del EFE en el caso planteado.

\subsubsection{Lista de los saldos que componen el Estado de Situación Financiera}

En la tabla 3 se exhiben los símbolos en letras que representan los valores correspondientes al Estado de Situación Financiera (ESF), para cada rubro.

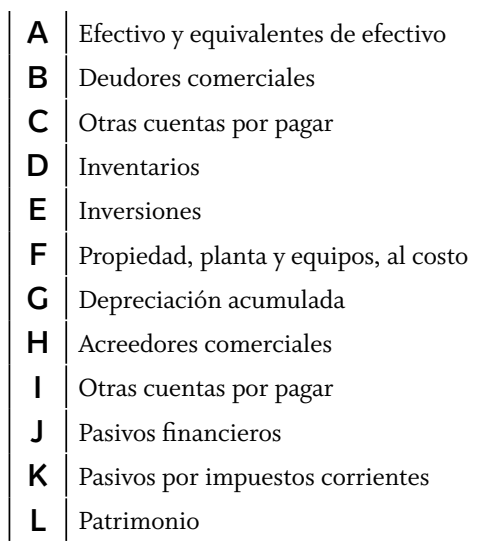

Tabla 3. Lista de símbolos utilizados como valores en el Estado de Situación Financiera, ESF

Fuente: elaboración propia

\subsubsection{Lista de los valores que} representan los valores incluidos en el Estado de Resultados y en los desgloses efectuados a las variaciones

En la tabla 4 se presentan los símbolos en letras que representan los valores incluidos en el Estado de Resultados, los desgloses de las variaciones del ESF y los valores de los diferentes conceptos por flujos de efectivo de las actividades de inversión y financiación, cuya información es reportada por las áreas de tesorería y/o gerencia financiera.

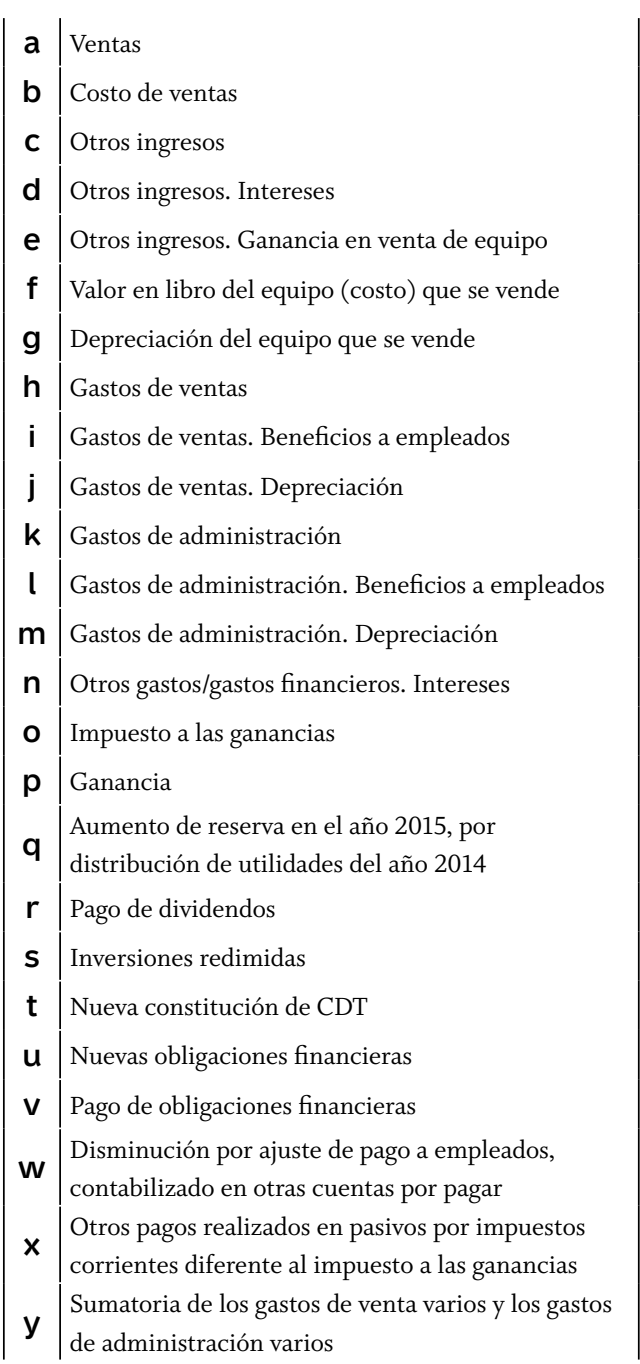

Tabla 4. Lista de símbolos utilizados en el Estado de Resultados y en los desgloses de conceptos Fuente: elaboración propia

\subsubsection{Lista de símbolos que representan} valores en totales y subtotales utilizados en los diferentes estados financieros

En la tabla 5 están los símbolos en letras utilizados para representar los valores de los totales y subtotales de los estados financieros. 


\begin{tabular}{|c|l|} 
Ac & Total de activos corrientes \\
Anc & Total de activos no corrientes \\
FneAF & $\begin{array}{l}\text { Faldo de capital } \\
\text { financiación }\end{array}$ \\
FneAl & $\begin{array}{l}\text { Flujo neto de efectivo de las actividades de } \\
\text { inversión }\end{array}$ \\
FneAO & $\begin{array}{l}\text { Flujo neto de efectivo de las actividades de } \\
\text { operación }\end{array}$ \\
Gai & Ganancia antes de impuestos \\
Gb & Ganancia bruta \\
Pc & Total de pasivos corrientes \\
Rs & Saldo de reserva \\
TA & Total de activos \\
TPP & Total de pasivo y patrimonio
\end{tabular}

Tabla 5. Lista de símbolos que representan valores en totales y subtotales

Fuente: elaboración propia

\subsection{Estados financieros}

A continuación, los tres estados financieros del caso planteado con los valores de manera simbólica, con letras estipuladas según las tablas 3 , 4 y 5 .

\subsubsection{Estado de Situación Financiera (ESF)}

El ESF de la empresa ADS comparativo al corte del 31 de diciembre de los años 2014 y 2015, se muestra en la tabla 6, no detalla el patrimonio, debido a que esto está en el respectivo Estado de Cambios del Patrimonio.

\section{Empresa ADS \\ Estado de Situación Financiera \\ Al 31 de diciembre de}

Activos

Activos corrientes

Efectivo y equivalentes de efectivo

Deudores comerciales

Otras cuentas por cobrar

$\mathrm{A}_{2} \quad \mathrm{~A}$

Inventarios

$\mathrm{B}_{2} \quad \mathrm{~B}_{1}$

$\mathrm{C}_{2} \quad \mathrm{C}_{1}$

$\mathrm{D}_{2} \quad \mathrm{D}_{1}$

Inversiones

Total activos corrientes

$\mathrm{E}_{2} \quad \mathrm{E}_{1}$

Activos no corrientes

Propiedad, planta y equipos, al costo

Depreciación acumulada

Total activos no corrientes

Total activos

$\mathrm{Ac}_{2} \quad \mathrm{Ac}_{1}$

$\begin{array}{cc}\mathrm{F}_{2} & \mathrm{~F}_{1} \\ \mathrm{G}_{2} & \mathrm{G}_{1} \\ \mathrm{Anc}_{2} & \mathrm{AnC}_{1} \\ \mathrm{TA} & \mathrm{TA}\end{array}$

Pasivos

Pasivos corrientes

Acreedores comerciales

Otras cuentas por pagar

Pasivos financieros

Pasivos por impuestos corrientes

Total pasivos corrientes

Total pasivos

Patrimonio

Total pasivo y patrimonio

$\begin{array}{cc}\mathrm{H}_{2} & \mathrm{H}_{1} \\ \mathrm{I}_{2} & \mathrm{I}_{1} \\ \mathrm{~J}_{2} & \mathrm{~J}_{1} \\ \mathrm{~K}_{2} & \mathrm{~K}_{1} \\ \mathrm{Pc}_{2} & \mathrm{Pc}_{1} \\ \mathrm{TP}_{2} & \mathrm{TP}_{1} \\ \mathrm{~L}_{2} & \mathrm{~L}_{1} \\ \mathrm{TPP}_{2} & \mathrm{TPP}_{1}\end{array}$

Tabla 6. Estado de Situación Financiera (ESF)

Fuente: elaboración propia

\subsubsection{Estado de Resultados}

El Estado de Resultados ${ }^{7}$ de la empresa ADS, dedicada a la actividad comercial, presentado en el formato “por función”, según NIC 1, párrafo 103, correspondiente al período del 1 de enero al 31 de diciembre del año 2015, se muestra en la tabla 7.

7 Incluye los gastos por impuestos conforme a lo establecido en el literal d del párrafo 82 de la NIC 1 . 
Empresa ADS

Estado de Resultados

Del 1 de enero al 31 de diciembre de 2015

\begin{tabular}{lc} 
Ventas & $+\mathrm{a}$ \\
Costo de ventas & $-\mathrm{b}$ \\
\cline { 2 - 2 } Ganancia bruta & $\mathrm{Gb}$ \\
Otros ingresos & $+\mathrm{c}$ \\
Gastos de venta & $-\mathrm{h}$ \\
Gastos de administración & $-\mathrm{k}$ \\
Otros gastos & $-\mathrm{n}$ \\
\hline Ganancia antes de impuestos & $\mathrm{Gai}$ \\
\hline Impuestos a las ganancias & $-\mathrm{o}$ \\
Ganancia & $+\mathrm{p}$ \\
\hline
\end{tabular}

Tabla 7. Estado de Resultados para el caso planteado

Fuente: elaboración propia

\subsubsection{Estado de Cambios en el \\ Patrimonio}

El Estado de Cambios en el Patrimonio del caso en estudio, correspondiente al período comprendido entre el 1 de enero al 31 de diciembre del año 2015, se muestra en la tabla ocho, en donde el cero se representa por el símbolo $\emptyset$.

\subsection{Políticas contables}

Las políticas contables de la empresa ADS con respecto a la presentación y revelación de la información del EFE son las siguientes:

- La presentación del EFE se hará por el método directo, clasificando los flujos de efectivo en actividades de operación, de inversión y de financiación.

- Los saldos de efectivo y equivalentes lo conforman los saldos de las cuentas de caja y banco, y las inversiones con plazo inferior a tres meses.

- Se excluyen del EFE las transacciones no monetarias (párrafo 43 y 44 de la NIC 7) que se identifiquen a lo largo del proceso de elaboración de EFE. Este proceso debe tener cuidado de revisar si se han registrado conceptos que no afectan el efectivo, como depreciaciones, provisiones, deterioros o de ajustes realizados que afectan alguna cuenta de resultados y que tengan como contrapartida cuentas del patrimonio, del pasivo y/o del activo diferentes al efectivo o sus equivalentes. Los montos significativos de estas

\section{Empresa ADS}

Estado de cambios en el patrimonio

Del 1 de enero al 31 de diciembre de 2015

\begin{tabular}{|c|c|c|c|c|c|}
\hline Conceptos & Capital & Reserva & Ganancia & $\begin{array}{c}\text { Ganancia } \\
\text { del período } \\
\text { anterior }\end{array}$ & Total \\
\hline Saldo al 31 de diciembre del año 2014 & $+\mathrm{Cp}$ & + Rs & $+q+r$ & $\varnothing$ & $\mathrm{L}_{1}$ \\
\hline Reclasificación ganancia del año 2014 & & & $-(q+r)$ & $+(q+r)$ & $\varnothing$ \\
\hline Distribución de ganancias del año 2014 & & $+q$ & & $-(q+r)$ & $-\mathrm{r}$ \\
\hline Registro de ganancias del año 2015 & & & $+\mathrm{p}$ & & $+\mathrm{p}$ \\
\hline Saldo al 31 de diciembre del año 2015 & $+\mathrm{Cp}$ & $+\mathrm{Rs}+\mathrm{q}$ & $+\mathrm{p}$ & $\varnothing$ & $\mathrm{L}_{2}$ \\
\hline
\end{tabular}

Tabla 8. Estado de Cambios en el Patrimonio para el caso planteado Fuente: elaboración propia 
transacciones no monetarias se revelarán en las correspondientes notas a los estados financieros.

- En caso de que la empresa tenga alguna restricción sobre el efectivo y sus equivalentes lo reportará en las respectivas notas a los estados financieros (párrafo 48 de la NIC 7).

- Los ajustes registrados durante el período contable por concepto de diferencia en cambio de efectivo en moneda extranjera por pérdidas o ganancias no realizadas, no se reportarán como flujos de efectivo y, se incluirán como ajuste en la conciliación de la variación del efectivo y equivalente resultante, para obtener el respectivo saldo final que se presenta en el Estado de Situación Financiera (párrafo 28 de la NIC7).

- Se revelarán por separado los flujos de efectivo por los conceptos de recaudo de intereses, pago de intereses, recaudo de dividendos, pago de dividendos, pago del impuesto a las ganancias. El monto de estos flujos de efectivo y aquellos reportados en las actividades de inversión y financiación deben coincidir con la información que reporta el área de gerencia financiera.

- Los flujos de efectivo que se relacionan con aquellas partidas que hacen parte de la determinación del resultado del ejercicio son clasificados en las actividades de operación, como intereses recibidos, pagos de intereses, dividendos recibidos, impuesto a las ganancias; esto teniendo en cuenta las opciones planteadas $^{8}$ en el párrafo 33 de la NIC 7.

8 Las opciones planteadas en el párrafo 33 de la NIC 7 consisten en que los intereses pagados pueden ser clasificados como actividades de operación o de financiación y, los
- Los demás flujos de efectivo que se incluyen en las actividades de operación, obtenidos de la información contable, serán por conceptos como recaudo a clientes, otros recaudos, pago a proveedores, pago a empleados y otros pagos.

- Los flujos de efectivo que se relacionan con aquellas cuentas del pasivo no corriente o del patrimonio son clasificados en las actividades de financiación, por ejemplo: dividendos pagados 9 (párrafo 34 de la NIC 7).

- La política contable para determinar los flujos de efectivo en las actividades del EFE, presentada en el gráfico 1, se resumen así:

- Las actividades de operación la conforman todas aquellas partidas que afectan el efectivo que se relacionan con el resultado del ejercicio, las variaciones del activo corriente sin incluir las inversiones, las variaciones del pasivo corriente sin incluir las obligaciones financieras. Las actividades de inversión la integran las partidas que afectan el efectivo que se relacionan con las variaciones del activo no corriente y de las cuentas de inversión clasificadas en el activo corriente. Las actividades de financiación incluyen las partidas que afectan el efectivo que se relacionan con las variaciones del pasivo no corriente, del patrimonio y de las cuentas de obligaciones financieras clasificadas en el pasivo corriente.

intereses y dividendos recibidos pueden ubicarse en las actividades de operación o de inversión.

9 Según el párrafo 34, los dividendos pagados pueden clasificarse como flujos de efectivo de actividades de financiación o de operación. 


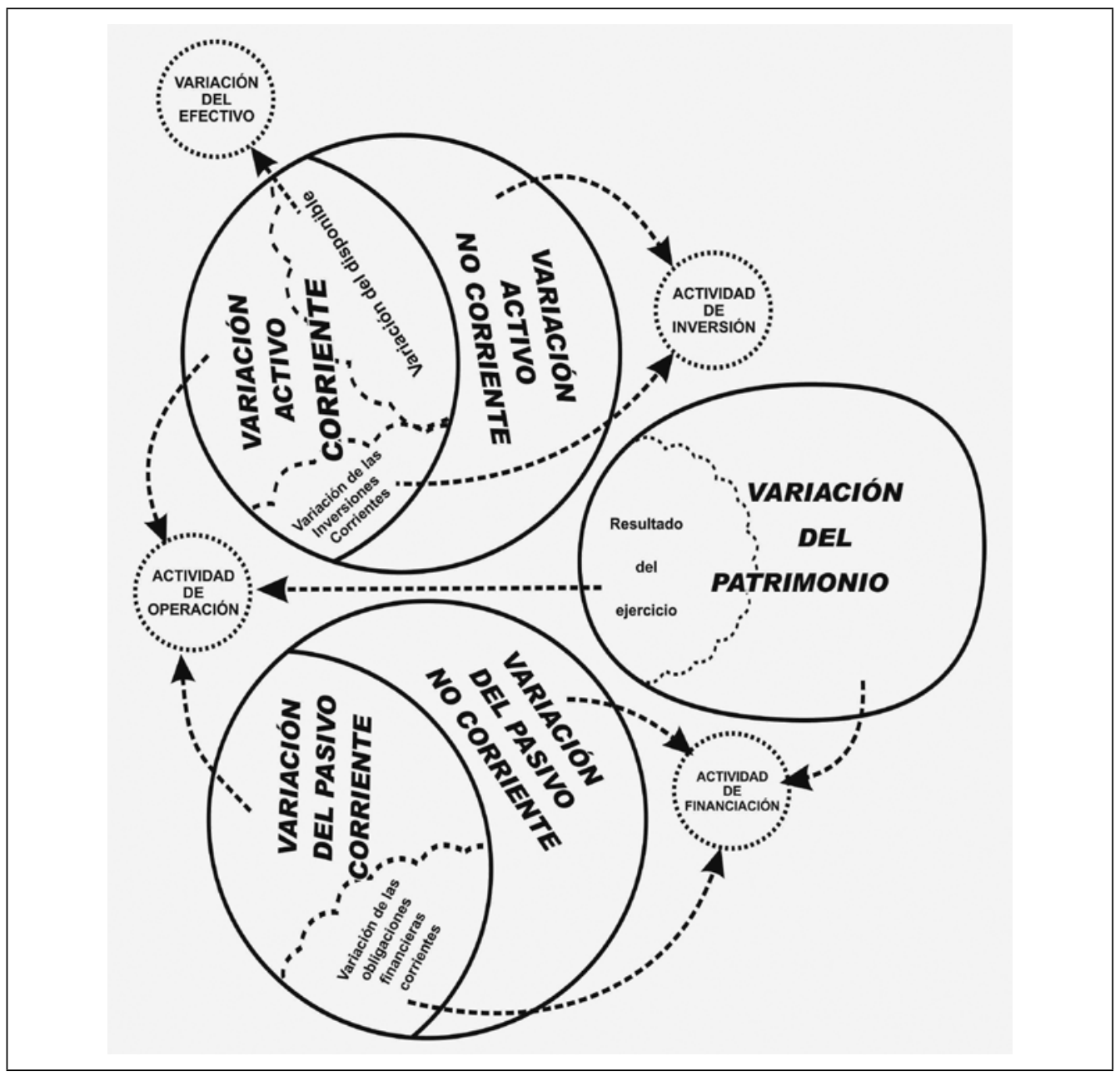

Gráfico 1. Determinación de los flujos de efectivo en las actividades del Estado de Flujos de Efectivo, EFE Fuente: elaboración propia

\subsection{Información adicional requerida}

La información adicional requerida es aquella que complementa la información ubicada en los otros tres estados financieros, la cual aparece en las notas a los estados financieros, en los auxiliares de las cuentas contables, en los registros contables y/o en información suministrada por las áreas de tesorería y de gerencia financiera.

\subsubsection{Variaciones del patrimonio}

Según el Estado de Cambios en el Patrimonio, la variación del patrimonio corresponde a la distribución de la ganancia del año 2014 y al registro de la ganancia del año 2015.

En los registros contables y en información suministrada por tesorería, se encuentra que en el mes de mayo de 2015 se pagaron a los accionistas el monto total de $\$ \mathrm{r}$, por concepto de 
pago de dividendos. Esto coincide con lo reflejado en el Estado de Cambios en el Patrimonio del período (ver tabla 8).

Entonces, el resumen de la variación de la cuenta de patrimonio es: $\$ \Delta \mathrm{L}=\mathrm{p}-\mathrm{r}$

\subsubsection{Variaciones del activo no corriente}

El activo no corriente está conformado por la cuenta de propiedad, planta y equipo, con su respectiva depreciación acumulada. Los registros identificados en estos rubros durante el año 2015 conciernen a la venta de un equipo en el mes de agosto, y a la depreciación del año 2015, según la revisión de los auxiliares de estas cuentas.

En cuanto al registro de la venta del equipo, se encontró que el costo en libros era de \$f y su depreciación acumulada hasta el mes de julio era de \$g, y registró una ganancia de \$e. El área de tesorería reportó que el recaudo por la venta del equipo fue recibido una parte en agosto y otra en septiembre.

Por lo anterior, las variaciones de las cuentas de propiedad, planta y equipo, al costo y la depreciación acumulada, se resumen así:

$\pm \Delta F+( \pm \Delta G)=-$ costo retirado del equipo vendido - depreciación del año 2015

\subsubsection{Variaciones de las cuentas de inversión}

El encargado del área de gerencia financiera reporta que durante el año 2015 las inversiones de la empresa ADS corresponden a CDT mayores a tres meses, que se resumen así:
El registro de las redenciones de CDT se contabiliza con crédito a la cuenta de inversiones y débito al banco. Por lo tanto, implica una disminución a la cuenta de inversiones y un aumento al efectivo. Por el contrario, la nueva constitución de CDT se registra con débito a la cuenta de inversiones y crédito al banco, que ocasiona un aumento a la cuenta de inversiones y una disminución al efectivo.

Con base en la información suministrada en el área de gerencia y a los registros contables, se verifica que los CDT redimidos a su vencimiento, durante el año 2015, no estuvieron registrados como equivalentes de efectivo durante el año 2014, y los nuevos CDT constituidos, que aparecen en la cuenta de inversiones a corte del 31 de diciembre de 2015, tienen un plazo mayor a tres meses para su redención.

El resumen de la variación de la cuenta de inversiones es: $\$ \Delta \mathrm{E}=\mathrm{t}-\mathrm{s}$

\subsubsection{Variaciones de las cuentas que contabilizan obligaciones financieras}

Las obligaciones financieras se contabilizaron en las cuentas de pasivos financieros y durante el año 2015, el área de gerencia financiera reportó lo siguiente:

Nuevas obligaciones financieras $\quad u$

El resumen de la variación de la cuenta de pasivos financieros es: $\$ \Delta \mathrm{J}=\mathrm{u}-\mathrm{v}$ 


\subsubsection{Gastos por depreciación, amortización y beneficios a los empleados}

Los gastos por depreciación, amortización y beneficios a los empleados se detallan en notas a los estados financieros de la empresa ADS; esto de acuerdo con la revelación que se estipula en el párrafo 104 de la NIC 1, por presentar el Estado de Resultados por función.

Total gastos por beneficio a empleados $=\$(i+l)$ Total gastos por depreciación $=\$(j+m)$

No tiene contabilizadas amortizaciones durante el año 2015.

Según la tabla 4, aparece el valor de \$y como la suma de los gastos de venta varios y los gastos de administración varios, que corresponden a la totalidad de los gastos de venta y administración restándoles los gastos por concepto de beneficios a empleados, de depreciación y de amortización, debido a que estos últimos requieren estar detallados, según la normativa contable anteriormente indicada.

\subsubsection{Detalle de otros ingresos}

El rubro de otros ingresos está conformado por:

d Otros ingresos. Intereses

e Otros ingresos. Ganancia en venta de equipo

c Total otros ingresos

Los $\$ \mathrm{~d}$ contabilizados como intereses surgen de los rendimientos obtenidos de los CDT. Se contabilizan con débito a la cuenta "Otras cuentas por cobrar", presentada en el Estado de Situación Financiera como activo corriente.

\subsubsection{Detalle de otros gastos}

Los otros gastos registrados durante el año 2015 por un total de $\$ \mathrm{n}$, corresponden a los intereses que se derivan de las obligaciones financieras y son contabilizados con crédito a la cuenta "Otras cuentas por pagar", clasificada en el Estado de Situación Financiera como pasivo corriente.

\subsubsection{Partidas no monetarias}

$\mathrm{Al}$ revisar los registros contables se encuentra que como partida no monetaria solamente se presenta la depreciación de propiedad, planta y equipo del período. No presenta provisiones ni amortizaciones.

Partidas no monetarias son aquellas que no afectan el efectivo, por lo general corresponden a registros contables que afectan cuentas del activo o del pasivo diferente al efectivo y equivalente de efectivo $y$, tienen como contrapartida las cuentas de resultados. Las partidas no monetarias más frecuentes son depreciación, amortización y provisión.

\subsubsection{Variación de efectivo y equivalentes de efectivo}

El rubro de efectivo y equivalente de efectivo para la empresa ADS lo conforman las cuentas de Caja y Bancos. No presenta efectivo en moneda extranjera. No presenta restricción sobre las partidas registradas como efectivo y equivalentes de efectivo. No tiene CDT a corte de 31 de diciembre de 2015 con vencimiento menor a tres meses.

Para el caso planteado, no es necesario conciliar los saldos reportados como efectivo y equivalentes de efectivo en el EFE y los saldos reportados en el ESF, debido a que en ambos estados financieros presentan los mismos saldos de efectivo y equivalentes. 


\subsubsection{Información complementaria}

Como complemento al análisis de las variaciones de las cuentas del ESF, aparte de lo anteriormente anotado, el caso plantea los siguientes asuntos relativos a la determinación de flujos de efectivos específicos:

- La empresa ha liquidado todos los beneficios a empleados a corte del 31 de diciembre de 2015, e incluso vacaciones e intereses de cesantías, por lo que todas las causaciones por acumulación, por este concepto, realizadas durante el año están debidamente pagadas al corte del año 2015.

- No tiene flujos de efectivo por concepto de dividendos recibidos.

- No presenta restricción sobre sus activos.

- No presenta transacciones en moneda extranjera.

\subsection{Proceso de elaboración del EFE del caso planteado}

\subsubsection{Fase 1. Conformación de la}

\section{ecuación preliminar de la variación} del efectivo y equivalentes

Esta primera fase consiste en transformar la doble ecuación del Estado de Situación Financiera (ESF) en una ecuación preliminar de la variación del efectivo y equivalentes de efectivo, expresada de manera vertical.

\subsubsection{Doble ecuación del ESF}

Esta tarea consiste en colocar la información del ESF comparativo a dos años consecutivos, en el formato de doble ecuación. Al frente del concepto de los rubros contables, se ubican las dos columnas con los valores de los saldos respectivos, que conforman la doble ecuación del ESF de forma vertical, identificada como ecuación central 1, como se muestra en la tabla 9.

\begin{tabular}{lcc} 
Efectivo y equivalentes de efectivo & $\mathrm{A}_{2}$ & $\mathrm{~A}_{1}$ \\
Deudores comerciales & $\mathrm{B}_{2}$ & $\mathrm{~B}_{1}$ \\
Otras cuentas por cobrar & $\mathrm{C}_{2}$ & $\mathrm{C}_{1}$ \\
Inventarios & $\mathrm{D}_{2}$ & $\mathrm{D}_{1}$ \\
Inversiones & $\mathrm{E}_{2}$ & $\mathrm{E}_{1}$ \\
Propiedad, planta y equipos, al costo & $\mathrm{F}_{2}$ & $\mathrm{~F}_{1}$ \\
Depreciación acumulada & $\mathrm{G}_{2}$ & $\mathrm{G}_{1}$ \\
\hline Pcreedores comerciales & & \\
Otras cuentas por pagar & $\mathrm{H}_{2}$ & $\mathrm{H}_{1}$ \\
Pasivos financieros & $\mathrm{I}_{2}$ & $\mathrm{I}_{1}$ \\
Pasivos por impuestos corrientes & $\mathrm{J}_{2}$ & $\mathrm{~J}_{1}$ \\
Patrimonio & $\mathrm{K}_{2}$ & $\mathrm{~K}_{1}$ \\
& $\mathrm{~L}_{2}$ & $\mathrm{~L}_{1}$
\end{tabular}

Tabla 9. Ecuación central 1

Fuente: elaboración propia

\subsubsection{Triple ecuación del ESF}

La triple ecuación del ESF se conforma al adicionar a la ecuación central 1 , la columna que corresponde a las variaciones de los respectivos saldos, que resulta de tomar el saldo del último período y restarle el saldo del período anterior. Se antepone el signo delta $\Delta$ para indicar la variación de cada rubro contable y se coloca \pm , debido a que cada variación tiene la posibilidad de ser positiva o negativa, dependiendo de si el saldo del último período es mayor o menor con respecto al saldo del período anterior. Esta triple ecuación del ESF se expresa en la ecuación central 2 que aparece en la tabla 10. 


$\begin{array}{llll}\text { Efectivo y equivalentes de efectivo } & \mathrm{A}_{2} & \mathrm{~A}_{1} & \pm \Delta \mathrm{A} \\ \text { Deudores comerciales } & \mathrm{B}_{2} & \mathrm{~B}_{1} & \pm \Delta \mathrm{B} \\ \text { Otras cuentas por cobrar } & \mathrm{C}_{2} & \mathrm{C}_{1} & \pm \Delta \mathrm{C} \\ \text { Inventarios } & \mathrm{D}_{2} & \mathrm{D}_{1} & \pm \Delta \mathrm{D} \\ \text { Inversiones } & \mathrm{E}_{2} & \mathrm{E}_{1} & \pm \Delta \mathrm{E} \\ \text { Propiedad, planta y equipos, al costo } & \mathrm{F}_{2} & \mathrm{~F}_{1} & \pm \Delta \mathrm{F} \\ \text { Depreciación acumulada } & \mathrm{G}_{2} & \mathrm{G}_{1} & \pm \Delta \mathrm{G}\end{array}$

$\begin{array}{llll}\text { Acreedores comerciales } & \mathrm{H}_{2} & \mathrm{H}_{1} & \pm \Delta \mathrm{H} \\ \text { Otras cuentas por pagar } & \mathrm{I}_{2} & \mathrm{I}_{1} & \pm \Delta \mathrm{I} \\ \text { Pasivos financieros } & \mathrm{J}_{2} & \mathrm{~J}_{1} & \pm \Delta \mathrm{J} \\ \text { Pasivos por impuestos corrientes } & \mathrm{K}_{2} & \mathrm{~K}_{1} & \pm \Delta \mathrm{K} \\ \text { Patrimonio } & \mathrm{L}_{2} & \mathrm{~L}_{1} & \pm \Delta \mathrm{L}\end{array}$

Tabla 10. Ecuación central 2

Fuente: elaboración propia

\subsubsection{Ecuación de las variaciones del ESF}

Se retiran las dos columnas de saldos a la ecuación central 2 y se incorpora la expresión "variación de" a cada rubro contable; con esto se conforma la ecuación central 3, como se indica en la tabla 11.

$\begin{array}{ll}\text { Variación del efectivo y equivalentes de efectivo } & \pm \Delta \mathrm{A} \\ \text { Variación de deudores comerciales } & \pm \Delta \mathrm{B} \\ \text { Variación de otras cuentas por cobrar } & \pm \Delta \mathrm{C} \\ \text { Variación de inventarios } & \pm \Delta \mathrm{D} \\ \text { Variación de inversiones } & \pm \Delta \mathrm{E} \\ \text { Variación de propiedad, planta y equipos, al costo } & \pm \Delta \mathrm{F} \\ \text { Variación de depreciación acumulada } & \pm \Delta \mathrm{G} \\ \end{array}$

Variación de acreedores comerciales

Variación de otras cuentas por pagar

$\pm \Delta \mathrm{I}$

Variación de pasivos financieros

$\pm \Delta \mathrm{J}$

Variación de pasivos por impuestos corrientes

$\pm \Delta \mathrm{K}$

Variación del patrimonio

$\pm \Delta \mathrm{L}$

\subsubsection{Despeje de las variaciones del} efectivo y equivalentes de efectivo

Con el propósito de ubicar la variación del efectivo y equivalentes en la parte inferior, para obtener la estructura de la ecuación del EFE (ver tabla 2); se realiza el despeje de esa variación colocándola en la parte de abajo. Entonces, al trasladar las variaciones del efectivo y equivalentes (que pasan del extremo superior al inferior), y cambiar el extremo donde se ubican las variaciones del pasivo y patrimonio (que pasan del extremo inferior al superior), con el fin de mantener la igualdad, se les debe cambiar el signo; y por tanto, se les antepone un signo negativo. Con esto se obtiene la ecuación central 4 en la tabla 12.

\begin{tabular}{ll} 
Variación de deudores comerciales & $\pm \Delta \mathrm{B}$ \\
Variación de otras cuentas por cobrar & $\pm \Delta \mathrm{C}$ \\
Variación de inventarios & $\pm \Delta \mathrm{D}$ \\
Variación de inversiones & $\pm \Delta \mathrm{E}$ \\
Variación de propiedad, planta y equipos, al & $\pm \Delta \mathrm{F}$ \\
costo & $\pm \Delta \mathrm{G}$ \\
Variación de depreciación acumulada & $-( \pm \Delta \mathrm{H})$ \\
Variación de acreedores comerciales & $-( \pm \Delta \mathrm{I})$ \\
Variación de otras cuentas por pagar & $-( \pm \Delta \mathrm{J})$ \\
Variación de pasivos financieros & $-( \pm \Delta \mathrm{K})$ \\
Variación de pasivos por impuestos corrientes & $-( \pm \Delta \mathrm{L})$ \\
Variación del patrimonio & \\
\hline & \\
\hline \hline & \\
Variación del efectivo y equivalentes de & $( \pm \Delta \mathrm{A})$ \\
\hline
\end{tabular}

Tabla 12. Ecuación central 4

Fuente: elaboración propia 


\subsubsection{Cambio de signos en ambos extremos}

La variación del efectivo y equivalentes que aparece en la ecuación central 4 por tener el signo negativo antepuesto expresa que se cambia el signo al resultado que se obtenga de la variación. Es decir, que si la variación resultara positiva (por aumento del efectivo y equivalentes) se le debería colocar en negativo y, si la variación resultara negativa, entonces, quedaría en positivo; lo que no es correcto porque este estado financiero muestra los flujos de efectivo en el sentido que les corresponda (con positivo, los aumentos y con negativo, las disminuciones del efectivo). Por lo tanto, se debe quitar ese signo negativo y conservar la igualdad; entonces, se debe multiplicar por -1 a ambos extremos. Con esto, se obtiene la ecuación preliminar del efectivo y equivalentes expresada en la ecuación central 5 que aparece en la tabla 13, y se termina la fase 1.

\begin{tabular}{lc} 
Variación de deudores comerciales & $-( \pm \Delta \mathrm{B})$ \\
Variación de otras cuentas por cobrar & $-( \pm \Delta \mathrm{C})$ \\
Variación de inventarios & $-( \pm \Delta \mathrm{D})$ \\
Variación de inversiones & $-( \pm \Delta \mathrm{E})$ \\
Variación de propiedad, planta y equipos, al & $-( \pm \Delta \mathrm{F})$ \\
costo & $-( \pm \Delta \mathrm{G})$ \\
Variación de depreciación acumulada & $\pm \Delta \mathrm{H}$ \\
Variación de acreedores comerciales & $\pm \Delta \mathrm{I}$ \\
Variación de otras cuentas por pagar & $\pm \Delta \mathrm{J}$ \\
Variación de pasivos financieros & $\pm \Delta \mathrm{K}$ \\
Variación de pasivos por impuestos corrientes & $\pm \Delta \mathrm{L}$ \\
Variación del patrimonio & \\
\hline \hline
\end{tabular}

Variación del efectivo y equivalentes de efectivo

Tabla 13. Ecuación central 5

Fuente: elaboración propia

\subsubsection{Fase 2. Depuración de la ecuación preliminar de la variación del efectivo $y$ equivalentes}

La fase de depuración de la ecuación preliminar de la variación del efectivo y equivalentes comprende el análisis riguroso que desglosa los componentes de ciertas variaciones que se indican más adelante, para luego identificar y/o determinar los flujos de efectivo allí inmersos.

Las variaciones que se van a analizar son las siguientes:

- Patrimonio (en dos partes: primero sin incluir el detalle la ganancia, y segundo se hace el desglose de esta)

- Activo no corriente

- Inversiones

- Pasivo no corriente (no aplica en este caso, porque no aparecen pasivos clasificados como no corrientes)

- Cuentas que contabilizan las obligaciones financieras, que para el caso planteado corresponden a los pasivos financieros

En esta segunda fase se determinan los flujos de efectivo de las actividades de inversión y de financiación, y otros conceptos, como resultado del análisis de variaciones. De manera que a medida que se va analizando cada variación, se establece la respectiva ecuación temporal, y se reemplaza su equivalente en la ecuación central. Al formar cada ecuación temporal, se debe procurar que inicie con lo favorable para el efectivo, para lograr una mejor presentación.

A continuación, se relacionan las variaciones depuradas de acuerdo con el caso planteado para elaborar el EFE. 


\subsubsection{Depuración de la variación del}

\section{patrimonio}

La depuración de la variación del patrimonio se logra tomando la información contenida en el Estado de Cambios en el Patrimonio del caso planteado. Específicamente, se toma la última columna del total, sin incluir los saldos, y se expresa en una ecuación temporal, así:

$\pm \Delta \mathrm{L}=-\mathrm{r}+\mathrm{p}$

Con los encargados de la gerencia financiera, se verifica que el pago de dividendos efectuado durante el año 2015 fue por \$r, que corresponde a la distribución de ganancias del año 2014, según la columna total del Estado de Cambios en el Patrimonio. Entonces, la ecuación temporal Et $\mathrm{t}_{1}$ indica que la variación del patrimonio (que puede ser positiva o negativa) es igual al pago de dividendos (negativo, porque disminuye el efectivo) más las ganancias del período (positiva, porque afecta con aumento el efectivo).

Para mejorar la presentación de la ecuación temporal $\mathrm{Et}_{1}$, de modo que inicie con lo favorable para el efectivo, se coloca primero el término expresado en positivo y luego el negativo, así:

$$
\pm \Delta \mathrm{L}=+\mathrm{p}-\mathrm{r}
$$

El equivalente de la variación del patrimonio, representada como “ $\pm \Delta \mathbf{L}$ ”, expresada en la ecuación $\mathrm{Et}_{2}$, se reemplaza en la ecuación central 5, con lo cual se obtiene la ecuación central 6, incluida en la tabla 14.

$\begin{array}{ll}\text { Variación de acreedores comerciales } & \pm \Delta \mathrm{H} \\ \text { Variación de otras cuentas por pagar } & \pm \Delta \mathrm{I} \\ \text { Variación de pasivos financieros } & \pm \Delta \mathrm{J} \\ \text { Variación de pasivos por impuestos corrientes } & \pm \Delta \mathrm{K} \\ \text { Ganancia } & +\mathrm{p} \\ \text { Pago de dividendos } & -\mathrm{r} \\ \text { Variación de deudores comerciales } & -( \pm \Delta \mathrm{B}) \\ \text { Variación de otras cuentas por cobrar } & -( \pm \Delta \mathrm{C}) \\ \text { Variación de inventarios } & -( \pm \Delta \mathrm{D}) \\ \text { Variación de inversiones } & -( \pm \Delta \mathrm{E}) \\ \text { Variación de propiedad, planta y equipos, al } & -( \pm \Delta \mathrm{F}) \\ \text { costo } & -( \pm \Delta \mathrm{G}) \\ \text { Variación de depreciación acumulada } & \end{array}$

Variación del efectivo y equivalentes de efectivo

Tabla 14. Ecuación central 6

Fuente: elaboración propia

\subsubsection{Desglose de la ganancia}

Esta tarea consiste en determinar y reemplazar, mediante una ecuación temporal, la equivalencia de la ganancia del período representada por "p". Para esto, el primer paso es determinar los componentes básicos del estado de resultado, con base en este estado financiero básico (ver tabla 7), que consiste en quitarle los subtítulos, con lo que se obtiene lo siguiente:

\begin{tabular}{lc} 
Ventas & $+\mathrm{a}$ \\
Costo de ventas & $-\mathrm{b}$ \\
Otros ingresos & $+\mathrm{c}$ \\
Gasto de ventas & $-\mathrm{h}$ \\
Gastos de administración & $-\mathrm{k}$ \\
Otros gastos & $-\mathrm{n}$ \\
Impuestos a las ganancias & $-\mathrm{o}$ \\
\hline Ganancia & $+\mathrm{p}$ \\
\hline
\end{tabular}


Con el detalle de los componentes básicos del estado de resultados se conforma la ecuación temporal $\mathrm{Et}_{3}$, así:

$a-b+c-h-k-n-o=p$

En la ecuación temporal $\mathrm{Et}_{3}$ se necesita desglosar la cuenta de otros ingresos, y determinar los conceptos de gastos por beneficios a empleados ${ }^{10}$ y por depreciación ${ }^{11}$. Esto se logra a partir de la información adicional obtenida, que se resume a continuación:

Detalle de otros ingresos \$c: Ingresos financieros. Intereses $\$ d$ Ganancias en venta de equipo e

Detalle de gastos de venta \$h:

Gastos por beneficios a empleados Gastos por depreciación

Gastos de venta varios

$$
\begin{aligned}
& \$ i \\
& j \\
& h-i-j
\end{aligned}
$$

Detalle de gastos de administración \$k:

Gastos por beneficios a empleados

Gastos por depreciación

$\mathrm{m}$

Gastos de administración varios

$\mathrm{k}-\mathrm{l}-\mathrm{m}$

Sumatoria de los gastos de venta varios y gastos de administración varios $=\$$ y

Con base en lo anterior, entonces, en primera instancia se obtienen las siguientes cuatro ecuaciones temporales:

$\mathrm{c}=\mathrm{d}+\mathrm{e}$

10 Para ir alistando la información que más adelante va a conformar el flujo de efectivo por concepto de pagos a empleados.

11 Para retirar las partidas (débito y crédito) por este concepto, porque no afectan el efectivo.

$$
\begin{aligned}
& -h=-(i+j+(h-i-j)) \\
& -k=-(l+m+(k-I-m)) \\
& y=+(h-i-j)+(k-I-m)
\end{aligned}
$$

Sumando las dos ecuaciones temporales $\mathrm{Et}_{5}$ y Et, $\mathrm{t}_{6}$, se obtiene:

$-h-k=-(i+j+(h-i-j))-(l+m+(k-I-m))$

$\mathrm{Al}$ organizar la ecuación temporal $\mathrm{Et}_{8}$ para expresarla en términos de $\mathrm{Et}_{7}$, se logra lo siguiente:

$$
-h-k=-(i+j+l+m)-((h-i-j)+(k-I-m))
$$

$\mathrm{Al}$ reemplazar $\mathrm{Et}_{7}$ en $\mathrm{Et}_{9}$, resulta la ecuación $\mathrm{Et}_{10}$, así:

$$
-h-k=-(i+j+l+m)-y
$$

Como los gastos por beneficios a empleados son: i, l y los gastos por depreciación son: j, m, entonces, se pueden reagrupar estos conceptos, así:

$$
-h-k=-(i+l)-(j+m)-y
$$

Con todo este análisis de las cuentas de resultado, se reemplazan los equivalentes de las ecuaciones temporales $\mathrm{Et}_{4}$ y Et $\mathrm{E}_{11}$ en $^{\mathrm{Et}} \mathrm{t}_{3}$ y se conforma $\mathrm{Et}_{12}$, así:

$$
a-b+d+e-(i+l)-(j+m)-y-n-o=p
$$


El equivalente de la ganancia, representada como "p" expresada en la ecuación Et ${ }_{12}$, se reemplaza en la ecuación central 6, teniendo en cuenta cambiar el concepto "Otros Gastos", por valor de $\$ \mathrm{n}$, por su equivalente que en el caso planteado es el de "Gastos financieros. Intereses", con lo cual se obtiene la ecuación central 7 incluida en la tabla 15.

\begin{tabular}{lc} 
Variación de acreedores comerciales & $\pm \Delta \mathrm{H}$ \\
Variación de otras cuentas por pagar & $\pm \Delta \mathrm{I}$ \\
Variación de pasivos financieros & $\pm \Delta \mathrm{J}$ \\
Variación de pasivos por impuestos corrientes & $\pm \Delta \mathrm{K}$ \\
Ventas & $+\mathrm{a}$ \\
Costo de ventas & $-\mathrm{b}$ \\
Ingresos financieros. Intereses & $+\mathrm{d}$ \\
Ganancia por venta de equipos & $+\mathrm{e}$ \\
Gasto por beneficios a empleados & $-(\mathrm{i}+\mathrm{l})$ \\
Gastos por depreciación & $-(\mathrm{j}+\mathrm{m})$ \\
Gastos varios de administración y ventas & $-\mathrm{y}$ \\
Gastos financieros. Intereses & $-\mathrm{n}$ \\
Impuestos a las ganancias & $-\mathrm{o}$ \\
Pago de dividendos & $-( \pm \Delta \mathrm{D})$ \\
Variación de deudores comerciales & $-\mathrm{r}$ \\
Variación de otras cuentas por cobrar & $-( \pm \Delta \mathrm{B})$ \\
Variación de inventarios & $-( \pm \Delta \mathrm{C})$ \\
Variación de inversiones & $-( \pm \Delta \mathrm{G})$ \\
Variación de propiedad, planta y equipos, al & \\
Vasto & \\
\hline \hline
\end{tabular}

Variación del efectivo y equivalentes de efectivo

\subsubsection{Depuración de las variaciones de las cuentas clasificadas como activos no corrientes}

Para el caso planteado, el activo no corriente comprende el costo de la propiedad, planta y equipo, y la depreciación acumulada.

Las variaciones de los rubros de propiedad, planta y equipos (al costo) y de depreciación acumulada, según la ecuación central 7, aparecen representadas como: $-( \pm \Delta \mathrm{F})$ y $-( \pm \Delta \mathrm{G})$, respectivamente; el signo negativo indica que se debe cambiar el signo que resulte del cálculo de la variación, que puede ser positiva o negativa. Esto es correcto porque la ecuación central 7 está en función del efectivo y no en función de la naturaleza de las cuentas.

Para facilitar el análisis a realizar, primero se analizan las variaciones antes de cambiarles el signo; es decir, cuando el signo coincidía con la naturaleza de las cuentas de propiedad, planta y equipo (al costo), que es débito, y por tanto, el aumento de las variaciones, en positivo, corresponde a registros débito, mientras que las disminuciones representan créditos, con variación negativa. Por el contrario, para el caso de las variaciones de depreciación acumulada, cuya naturaleza de las cuentas es opuesta a la de los activos: es crédito; entonces, los aumentos corresponden a registros créditos, representados con el signo negativo, y las disminuciones son registros débitos, representados con variación positiva.

- Detalle de la variación de propiedad, planta y equipo, costo, representado como $\pm \Delta \mathrm{F}$ 
Para el caso específico planteado, solamente varía la cuenta por concepto de disminución por venta de equipo (con ganancia), que por ser registro crédito, se representa negativamente: $-\mathrm{f}$

- Desglose de la variación de la depreciación acumulada, representada por: $\pm \Delta \mathrm{G}$ :

Disminución por la depreciación del equipo que se vende, es débito $+g$

Aumento por la depreciación del año, es crédito

Total variación de depreciación acumulada

$\frac{-(j+m)}{ \pm \Delta G}$

Luego, se suman las dos variaciones de propiedad, planta y equipo al costo y depreciación acumulada, para iniciar el análisis que determinará los flujos de efectivo y demás aspectos que lo componen. Esto conforma la ecuación temporal 13, así:

$\pm \Delta F+( \pm \Delta G)=-f+g-(j+m)$

Seguidamente, se cambian los signos en ambos extremos de la ecuación, para expresar como indica la figura 11, los signos negativos de las variaciones de propiedad, planta y equipos (costo) y de la depreciación acumulada.

$-(( \pm \Delta F)+( \pm \Delta G))=-(-f+g-(j+m)) \quad\left(E_{14}\right)$

Se realiza el cambio de signos indicado en el extremo derecho de la ecuación temporal 5, para quitar los paréntesis, con lo cual quedaría la nueva ecuación temporal, así:

$-( \pm \Delta \mathrm{F})-( \pm \Delta \mathrm{G})=\mathrm{f}-\mathrm{g}+(\mathrm{j}+\mathrm{m})$

Teniendo en cuenta que el recaudo por venta de equipo es igual al costo del equipo menos la depreciación acumulada, que tiene el equipo, más la ganancia en venta del equipo. Entonces, expresado ese recaudo en términos de la información obtenida en la depuración del costo de propiedad, planta y equipo, y la depreciación acumulada, quedaría así:

Costo del equipo (valor en libros)

f

Menos: depreciación del equipo que se vende

$-g$

Más: ganancia en la venta del equipo

Igual: recaudo por venta de equipo

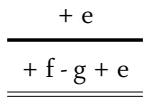

Por lo anterior, para el caso particular planteado, entonces, la ecuación del flujo de efectivo generado por la venta del equipo quedaría así:

Recaudo por venta de equipo $=\mathrm{f}-\mathrm{g}+\mathrm{e} \quad\left(\mathrm{Et}_{16}\right)$

Siguiendo con el análisis, al comparar las dos últimas ecuaciones temporales, $\mathrm{Et}_{15} \mathrm{y} \mathrm{Et}_{16}$, se observa que tienen en común lo siguiente: f - g

Entonces, se hace una sustitución de la expresión de una ecuación en la otra y como se pretende llegar a la variación de las cuentas propiedad, planta y equipos (incluyendo depreciación acumulada), se debe despejar en la ecuación $\mathrm{Et}_{16}$ la expresión $\mathrm{f}-\mathrm{g}$, con lo cual quedaría así:

Recaudo por venta de equipo - e = f - g $\quad\left(E_{17}\right)$

Ahora, la ecuación $\mathrm{Et}_{15}$ se ubica en la ecuación $\mathrm{Et}_{17}$, sustituyendo el equivalente de f-g, así:

- $( \pm \Delta \mathrm{F})-( \pm \Delta \mathrm{G})=$ Recaudo por venta de equipo $-e+(j+m)$ 
Entonces, para representar la ecuación con los símbolos, y no con los conceptos, se reemplaza el equivalente del Recaudo por venta de equipo, expresado en la ecuación temporal $16\left(\mathrm{Et}_{16}\right)$, así:

$-( \pm \Delta \mathrm{F})-( \pm \Delta \mathrm{G})=(\mathrm{f}-\mathrm{g}+\mathrm{e})-\mathrm{e}+(\mathrm{j}+\mathrm{m})$

El equivalente de las variaciones de propiedad, planta y equipos (al costo) y de depreciación acumulada, representadas como: $-( \pm \Delta \mathrm{F})$ y $-( \pm \Delta \mathrm{G})$, expresadas en la ecuación $\mathrm{Et}_{19}$, se reemplaza en la ecuación central 7, con lo cual se obtiene la ecuación central 8, incluida en la tabla 16.

Variación de acreedores comerciales
Variación de otras cuentas por pagar
Variación de pasivos financieros
Variación de pasivos por impuestos
corrientes
Ventas
Costo de ventas
Ingresos financieros. Intereses
Ganancia por venta de equipos
Gasto por beneficios a empleados
Gastos por depreciación
Gastos varios de administración y ventas
Gastos financieros. Intereses
Impuestos a las ganancias
Pago de dividendos
Variación de deudores comerciales
Variación de tras cuentas por cobrar
Variación de inventarios
Variación de inversiones
Recaudo por venta de equipo
Ganancia en venta de equipo
Depreciación acumulada

Variación del efectivo y equivalentes de efectivo

- e

$+(j+m)$

\subsubsection{Depuración de las variaciones de las cuentas de inversión}

Según la ecuación central 8, las variaciones de las cuentas de inversión están representadas como: - $( \pm \Delta E)$. Por lo tanto, en forma similar como se indicó al inicio del análisis de las variaciones de las cuentas del activo no corriente, se recomienda, en primera instancia, revisar las variaciones de inversión sin el cambio del signo, así: $\pm \Delta \mathrm{E}$.

Se analizan las variaciones de las cuentas de inversión (que no fueron incluidas como equivalentes de efectivo), conforme a la variación de la cuenta, es decir, según su naturaleza; los aumentos de las cuentas de inversiones se muestran con signo positivo y con signo negativo, las respectivas disminuciones. Entonces, según el caso estudiado, las variaciones corresponden al siguiente desglose:

\begin{tabular}{ll} 
Inversiones redimidas & $-\mathrm{s}$ \\
Nueva constitución de CDT & $+\mathrm{t}$ \\
\cline { 2 - 2 } Total variación de inversiones & $\pm \Delta \mathrm{E}$
\end{tabular}

Esto corresponde a la siguiente ecuación:

$$
\pm \Delta \mathrm{E}=-\mathrm{s}+\mathrm{t}
$$

$-( \pm \Delta \mathrm{E})=\mathrm{s}-\mathrm{t}$

El equivalente de la variación de inversiones, representada como “- $( \pm \Delta \mathbf{E})$ ”, expresada en $\mathrm{Et}_{2,}$, se reemplaza en la ecuación central 8, con 
lo cual se obtiene la ecuación central 9, incluida en la tabla 17.

\begin{tabular}{ll} 
Variación de acreedores comerciales & $\pm \Delta \mathrm{H}$ \\
Variación de otras cuentas por pagar & $\pm \Delta \mathrm{I}$ \\
Variación de pasivos financieros & $\pm \Delta \mathrm{J}$ \\
Variación de pasivos por impuestos & $\pm \Delta \mathrm{K}$ \\
corrientes & $+\mathrm{a}$ \\
Ventas & $-\mathrm{b}$ \\
Costo de ventas & $+\mathrm{d}$ \\
Ingresos financieros. Intereses & $+\mathrm{e}$ \\
Ganancia por venta de equipos & $-(\mathrm{i}+\mathrm{l})$ \\
Gasto por beneficios a empleados & $-(\mathrm{j}+\mathrm{m})$ \\
Gastos por depreciación & $-\mathrm{y}$ \\
Gastos varios de administración y ventas & $-\mathrm{n}$ \\
Gastos financieros. Intereses & $-\mathrm{o}$ \\
Impuestos a las ganancias & $-\mathrm{r}$ \\
Pago de dividendos & $-( \pm \Delta \mathrm{B})$ \\
Variación de deudores comerciales & $-( \pm \Delta \mathrm{C})$ \\
Variación de otras cuentas por cobrar & $-( \pm \Delta \mathrm{D})$ \\
Variación de inventarios & $+\mathrm{s}$ \\
Inversiones redimidas & $-\mathrm{t}$ \\
Nueva constitución de CDT & $+(\mathrm{f}-\mathrm{g}+\mathrm{e})$ \\
Recaudo por venta de equipo & $-\mathrm{e}$ \\
Ganancia en venta de equipo & $+(\mathrm{j}+\mathrm{m})$ \\
Depreciación acumulada & \\
\hline \hline
\end{tabular}

Variación del efectivo y equivalentes de efectivo

Tabla 17. Ecuación central 9

Fuente: elaboración propia

\subsubsection{Depuración de la variación de los}

\section{pasivos financieros}

Para el caso planteado, las variaciones de los pasivos financieros corresponde a:

Nuevas obligaciones financieras

Pago de obligaciones financieras

Total variación de pasivos financieros
Con esto se obtiene la siguiente ecuación temporal:

$$
\pm \Delta \mathrm{J}=+\mathrm{u}-\mathrm{v}
$$

El equivalente de la variación de pasivos financieros, representada como “ $\pm \Delta \mathrm{J}$ ”, expresada en la ecuación $\mathrm{Et}_{22}$, se reemplaza en la ecuación central 9, con lo cual se obtiene la ecuación central 10, incluida en la tabla 18.

$\begin{array}{lc}\text { Variación de acreedores comerciales } & \pm \Delta \mathrm{H} \\ \text { Variación de otras cuentas por pagar } & \pm \Delta \mathrm{I} \\ \text { Nuevas obligaciones financieras } & +\mathrm{u} \\ \text { Pago de obligaciones financieras } & -\mathrm{v} \\ \text { Variación de pasivos por impuestos } & \pm \Delta \mathrm{K} \\ \text { corrientes } & +\mathrm{a} \\ \text { Ventas } & -\mathrm{b} \\ \text { Costo de ventas } & +\mathrm{d} \\ \text { Ingresos financieros. Intereses } & +\mathrm{e} \\ \text { Ganancia por venta de equipos } & -(\mathrm{i}+\mathrm{l}) \\ \text { Gasto por beneficios a empleados } & -(\mathrm{j}+\mathrm{m}) \\ \text { Gastos por depreciación } & -\mathrm{y} \\ \text { Gastos varios de administración y ventas } & -\mathrm{n} \\ \text { Gastos financieros. Intereses } & -\mathrm{o} \\ \text { Impuestos a las ganancias } & -\mathrm{r} \\ \text { Pago de dividendos } & -( \pm \Delta \mathrm{B}) \\ \text { Variación de deudores comerciales } & -( \pm \Delta \mathrm{C}) \\ \text { Variación de otras cuentas por cobrar } & -( \pm \Delta \mathrm{D}) \\ \text { Variación de inventarios } & +\mathrm{s} \\ \text { Inversiones redimidas } & -\mathrm{f}-\mathrm{g}+\mathrm{e}) \\ \text { Nueva constitución de CDT } & \mathrm{j}+\mathrm{m}) \\ \text { Recaudo por venta de equipo } & \\ \text { Ganancia en venta de equipo } & \\ \text { Depreciación acumulada } & \\ & \end{array}$

Variación del efectivo y equivalentes de efectivo

Tabla 18. Ecuación central 10

Fuente: elaboración propia 


\subsubsection{Fase 3. Preparación de la} ecuación del EFE por el método directo

En esta fase se organizan los diferentes grupos por conceptos asignados previamente, los cuales identifican los diferentes flujos de las actividades de inversión y de financiación, el alistamiento para las preparaciones de los diferentes flujos de efectivo de la actividad de operación y las eliminaciones entre sí de partidas que no afectan el efectivo. Finalmente, se evalúa lo correcto de los montos calculados de los flujos de operación.

\subsubsection{Identificación de cada ítem en} actividades del EFE o que estén por eliminar (por no afectar el efectivo y equivalentes)

Se examina cada uno de los ítems ubicados en el extremo superior de la ecuación central 10, y de acuerdo a los criterios establecidos en las políticas contables de la empresa ADS, se procede a realizar lo siguiente:

- Análisis de los flujos de efectivo, que se rotulan en actividades del EFE de inversión o de financiación.

- Identificación de aquellas partidas, con signo positivo y negativo, que se deben eliminar por no afectar el efectivo y equivalentes. $\mathrm{Al}$ eliminarse no afectarán la equivalencia de la ecuación central, debido a que la suma algebraica entre ellas es igual a cero; para su control se enumera cada eliminación entre sí.

- Las demás partidas se marcan como actividad de operación, para posteriormente conformar los flujos de efectivo propios del método directo. Es necesario advertir que en el caso planteado, estas partidas coindicen con las variaciones no depuradas del Estado de Situación Financiera. Pero podrían presentarse casos en que se depuren estas variaciones; por ejemplo, si hubiera provisiones de inventario, entonces, la variación de inventario estaría depurada al separar de la variación inicial el efecto de la provisión, que constituye una partida a eliminar, que se retiraría con la respectiva cuenta de gastos.

En el caso planteado se obtiene lo que aparece en la tabla 19, que es la misma ecuación central 10 a la que se le agregaron la identificación de las actividades del EFE y las partidas a eliminar.

\subsubsection{Retiro de ítems identificados para eliminar, y agrupación por las actividades del EFE}

Se retiran los ítems identificados a eliminar. Lo restante se agrupa, ordenándolo por actividades de operación, inversión y financiación, con lo cual se obtiene la ecuación central 11 que se muestra en la tabla 20.

\subsubsection{Identificación y asignación para} conformar flujos de efectivo de la actividad de operación

A los ítems identificados como actividad de operación se les asigna la denominación del flujo de efectivo a que corresponden, según lo estipulado en las respectivas políticas contables de la empresa ADS, obteniendo lo que aparece en la tabla 21. 


\section{Clasificación}

\begin{tabular}{|c|c|c|}
\hline Variación de acreedores comerciales & $\pm \Delta \mathrm{H}$ & Actividad de operación \\
\hline Variación de otras cuentas por pagar & $\pm \Delta \mathrm{I}$ & Actividad de operación \\
\hline Nuevas obligaciones financieras & $+\mathrm{u}$ & Actividad de financiación \\
\hline Pago de obligaciones financieras & $-\mathrm{v}$ & Actividad de financiación \\
\hline Variación de pasivos por impuestos corrientes & $\pm \Delta \mathrm{K}$ & Actividad de operación \\
\hline Ventas & $+a$ & Actividad de operación \\
\hline Costo de ventas & $-b$ & Actividad de operación \\
\hline Ingresos financieros. Intereses & $+d$ & Actividad de operación \\
\hline Ganancia por venta de equipos & $+\mathrm{e}$ & Eliminar (No. 1) \\
\hline Gasto por beneficios a empleados & $-(\mathrm{i}+1)$ & Actividad de operación \\
\hline Gastos por depreciación & $-(j+m)$ & Eliminar (No. 2) \\
\hline Gastos varios de administración y ventas & $-y$ & Actividad de operación \\
\hline Gastos financieros. Intereses & $-\mathrm{n}$ & Actividad de operación \\
\hline Impuestos a las ganancias & -0 & Actividad de operación \\
\hline Pago de dividendos & $-\mathrm{r}$ & Actividad de financiación \\
\hline Variación de deudores comerciales & $-( \pm \Delta \mathrm{B})$ & Actividad de operación \\
\hline Variación de otras cuentas por cobrar & $-( \pm \Delta C)$ & Actividad de operación \\
\hline Variación de inventarios & $-( \pm \Delta \mathrm{D})$ & Actividad de operación \\
\hline Inversiones redimidas & $+\mathrm{s}$ & Actividad de inversión \\
\hline Nueva constitución de CDT & $-\mathrm{t}$ & Actividad de inversión \\
\hline Recaudo por venta de equipo & $+(f-g+e)$ & Actividad de inversión \\
\hline Ganancia en venta de equipo & $-\mathrm{e}$ & Eliminar (No. 1) \\
\hline Depreciación acumulada & $+(j+m)$ & Eliminar (No. 2) \\
\hline
\end{tabular}

Variación del efectivo y equivalentes de efectivo $\quad \pm \Delta \mathrm{A}$

Tabla 19. Ecuación central 10 con identificación de las actividades del EFE y las partidas a eliminar Fuente: elaboración propia 


\section{Actividad}

\begin{tabular}{|c|c|c|}
\hline Variación de acreedores comerciales & $\pm \Delta \mathrm{H}$ & Operación \\
\hline Variación de otras cuentas por pagar & $\pm \Delta \mathrm{I}$ & Operación \\
\hline Variación de pasivos por impuestos corrientes & $\pm \Delta \mathrm{K}$ & Operación \\
\hline Ventas & $+\mathrm{a}$ & Operación \\
\hline Costo de ventas & $-b$ & Operación \\
\hline Ingresos financieros. Intereses & $+\mathrm{d}$ & Operación \\
\hline Gasto por beneficios a empleados & $-(i+1)$ & Operación \\
\hline Gastos varios de administración y ventas & $-y$ & Operación \\
\hline Gastos financieros. Intereses & $-\mathrm{n}$ & Operación \\
\hline Impuestos a las ganancias & -0 & Operación \\
\hline Variación de deudores comerciales & $-( \pm \Delta \mathrm{B})$ & Operación \\
\hline Variación de otras cuentas por cobrar & $-( \pm \Delta C)$ & Operación \\
\hline Variación de inventarios & $-( \pm \Delta \mathrm{D})$ & Operación \\
\hline Inversiones redimidas & $+\mathrm{s}$ & Inversión \\
\hline Nueva constitución de CDT & $-\mathrm{t}$ & Inversión \\
\hline Recaudo por venta de equipo & $+(f-g+e)$ & Inversión \\
\hline Nuevas obligaciones financieras & $+\mathrm{u}$ & Financiación \\
\hline Pago de obligaciones financieras & $-\mathrm{v}$ & Financiación \\
\hline Pago de dividendos & $-r$ & Financiación \\
\hline
\end{tabular}

Variación del efectivo y equivalentes de efectivo $\quad \pm \Delta \mathrm{A}$

Tabla 20. Ecuación central 11

Fuente: elaboración propia

\subsubsection{Agrupación de ítems para conformar} los flujos de efectivo de la actividad de operación

Se agrupan los flujos de efectivo de la actividad de operación obtenidos en el paso anterior, y se ubican con el siguiente orden: recaudo de clientes, pago a proveedores, pago a empleados, recaudo de intereses, pago de intereses, pago de impuesto sobre las ganancias, otros pagos realizados, con lo cual se obtiene la ecuación central 12 y quedan presentados como aparece en la tabla 22. 


\begin{tabular}{ll} 
Variación de acreedores comerciales & $\pm \Delta \mathrm{H}$ \\
Variación de otras cuentas por pagar & $\pm \Delta \mathrm{I}$ \\
Variación de pasivos por impuestos corrientes & $\pm \Delta \mathrm{K}$ \\
Ventas & $+\mathrm{a}$ \\
Costo de ventas & $-\mathrm{b}$ \\
Ingresos financieros. Intereses & $+\mathrm{d}$ \\
Gasto por beneficios a empleados & $-(\mathrm{i}+\mathrm{l})$ \\
Gastos varios de administración y ventas & $-\mathrm{y}$ \\
Gastos financieros. Intereses & $-\mathrm{n}$ \\
Impuestos a las ganancias & $-\mathrm{o}$ \\
Variación de deudores comerciales & $-( \pm \Delta \mathrm{B})$ \\
Variación de otras cuentas por cobrar & $-( \pm \Delta \mathrm{C})$ \\
Variación de inventarios & $-( \pm \Delta \mathrm{D})$ \\
Inversiones redimidas & $+\mathrm{s}$ \\
Nueva constitución de CDT & $-\mathrm{t}$ \\
Recaudo por venta de equipo & $-\mathrm{v}$ \\
Nuevas obligaciones financieras & \\
\hline \hline
\end{tabular}

\section{Para conformar flujos de efectivo en actividad de operación}

Pago a proveedores

Otros pagos

Pago impuesto sobre las ganancias

Recaudo de clientes

Pago a proveedores

Recaudo de intereses

Pago a empleados

Otros pagos

Pago de intereses

Pago impuesto sobre las ganancias

Recaudo de clientes

Pago de intereses

Pago a proveedores

Actividad de inversión

Actividad de inversión

Actividad de inversión

Actividad de financiación

Actividad de financiación

Actividad de financiación

Variación del efectivo y equivalentes de efectivo $\pm \Delta \mathrm{A}$

Tabla 21. Ecuación central 11 con asignación de los flujos específicos de la actividad de operación Fuente: elaboración propia

\subsubsection{Determinación de ecuaciones} temporales de los flujos de efectivo en actividad de operación

Con base en la agrupación de flujos de efectivo específicos obtenidos en la actividad de operación, se plantea el cálculo de respectivas ecuaciones temporales. Luego, se verifican los datos con que cuente la entidad, y en caso de que no sean los correctos, se efectúan los ajustes necesarios, analizando rigurosamente las partidas y contrapartidas que intervienen en la determinación de los respectivos flujos de efectivo.

Este tipo de verificación hace que la realización del EFE constituya un análisis riguroso de la información contable de la empresa. 


\begin{tabular}{|c|c|c|}
\hline Ventas & $+\mathrm{a}$ & Recaudo de clientes \\
\hline Variación de deudores comerciales & $-( \pm \Delta \mathrm{B})$ & Recaudo de clientes \\
\hline Variación de acreedores comerciales & $\pm \Delta \mathrm{H}$ & Pago a proveedores \\
\hline Costo de ventas & $-b$ & Pago a proveedores \\
\hline Variación de inventarios & $-( \pm \Delta \mathrm{D})$ & Pago a proveedores \\
\hline Gasto por beneficios a empleados & $-(i+1)$ & Pago a empleados \\
\hline Ingresos financieros. Intereses & $+\mathrm{d}$ & Recaudo de intereses \\
\hline Variación de otras cuentas por cobrar & $-( \pm \Delta \mathrm{C})$ & Recaudo de intereses \\
\hline Variación de otras cuentas por pagar & $\pm \Delta \mathrm{I}$ & Pago de intereses \\
\hline Gastos financieros. Intereses & $-\mathrm{n}$ & Pago de intereses \\
\hline Variación de pasivos por impuestos corrientes & $\pm \Delta \mathrm{K}$ & Pago impuesto sobre las ganancias \\
\hline Impuestos a las ganancias & -0 & Pago impuesto sobre las ganancias \\
\hline Gastos varios de administración y ventas & $-y$ & Otros pagos \\
\hline Inversiones redimidas & $+\mathrm{s}$ & Actividad de inversión \\
\hline Nueva constitución de CDT & $-\mathrm{t}$ & Actividad de inversión \\
\hline Recaudo por venta de equipo & $+(f-g+e)$ & Actividad de inversión \\
\hline Nuevas obligaciones financieras & $+\mathrm{u}$ & Actividad de financiación \\
\hline Pago de obligaciones financieras & $-\mathrm{v}$ & Actividad de financiación \\
\hline Pago de dividendos & $-\mathrm{r}$ & Actividad de financiación \\
\hline
\end{tabular}

Tabla 22. Ecuación central 12

Fuente: elaboración propia

A continuación, las ecuaciones temporales obtenidas con base en la agrupación de los flujos de efectivo específicos de la actividad de operación, de acuerdo a lo indicado en la tabla 22. Recaudo de clientes $=\mathrm{a}-( \pm \Delta \mathrm{B})$ Pago a proveedores $= \pm \Delta \mathrm{H}-\mathrm{b}-( \pm \Delta \mathrm{D})\left(\mathrm{Et}_{24}\right)$ Pago a empleados $=-(\mathrm{i}+\mathrm{l})$

Recaudo de intereses $=\mathrm{d}-( \pm \Delta \mathrm{C})$

Pago de intereses $= \pm \Delta \mathrm{I}-\mathrm{k}$

Pago de impuesto sobre las ganancias $= \pm \Delta \mathrm{K}-\mathrm{o}$

Otros pagos $=-\mathrm{y}$

\section{Agrupación para conformar flujos de efectivo en actividad de operación}

$+\mathrm{a}$

$\Delta \mathrm{B})$

$-( \pm \Delta \mathrm{D})$

$( \pm \Delta \mathrm{C})$

$\pm \Delta \mathrm{I}$

$\pm \Delta \mathrm{K}$

$-0$

y

$+(f-g+e)$

$+\mathrm{u}$

\subsubsection{Revisión y ajuste de los flujos de efectivo de la actividad de operación}

Para el caso planteado, como resultado de confrontar la información disponible en la empresa ADS, suministrada por la gerencia financiera, con los flujos de efectivo determinados en la actividad de operación, se evidencian inconsistencias en los flujos de efectivo por concepto de pago de intereses, y de pago de impuesto sobre las ganancias, determinados según las ecuaciones $\mathrm{Et}_{27} \mathrm{y} \mathrm{Et}_{28}$, respectivamente. 
$\mathrm{Al}$ analizar rigurosamente los conceptos y componentes de las dos ecuaciones con inconsistencias, se ha evidenciado lo siguiente:

- En la ecuación temporal $\mathrm{Et}_{18}$, que determina los pagos de intereses, el monto de la variación de otras cuentas por pagar, representado por " $\Delta \mathrm{I}$ ", incluye un concepto que se relaciona con pagos $^{12}$ a empleados por un total de \$w. Por lo tanto, esto implica que se deben corregir los montos totales de los pagos a empleados y de pago de intereses, y se deben ajustar las respectivas ecuaciones temporales $\left(E_{25} y_{E t_{27}}\right)$ y se generan nuevas ecuaciones temporales, como se muestra a continuación:

Pago a empleados $=-(\mathrm{i}+\mathrm{l})-\mathrm{w}$

Pago de intereses $= \pm \Delta \mathrm{I}-\mathrm{k}+\mathrm{w}$

- En la ecuación temporal $\mathrm{Et}_{28}$, que determina el flujo de efectivo del Pago de impuesto sobre las ganancias, el monto de la variación de pasivos por impuestos corrientes, representado por " $\Delta \mathrm{K}$ ”, incluye pagos de impuestos diferentes al de impuesto sobre las ganancias, por un total \$t y, al compararlo con los flujos determinados anteriormente, se considera pertinente incluirlo en el flujo por concepto de otros pagos; por lo cual se deben ajustar los dos flujos (pago de impuesto sobre las ganancias y otros pagos) y sus respectivas ecuaciones temporales:

Pago de impuesto sobre las ganancias $= \pm \Delta \mathrm{K}$ $-\mathrm{o}+\mathrm{t}$

Otros pagos $=-\mathrm{y}-\mathrm{t}$

12 Los pagos se realizaron en 2015 y el registro del pasivo se había registrado en 2014.

\subsubsection{Conformación definitiva de los flujos de efectivo de la actividad de operación}

Se colocan los conceptos de los flujos de efectivo y sus equivalentes, reemplazando las ecuaciones temporales $\mathrm{Et}_{23}, \mathrm{Et}_{24} . \mathrm{Et}_{25}, \mathrm{Et}_{26}, \mathrm{Et}_{31}, \mathrm{Et}_{32}$ y Et $t_{33}$ en la ecuación central 12 con lo que se obtiene la ecuación central 13 que es la ecuación del EFE por el método directo, con la que se termina la fase 3; ver tabla 23.

\begin{tabular}{lc} 
Recaudo de clientes & $+\mathrm{a}-( \pm \Delta \mathrm{B})$ \\
Pago a proveedores & $\pm \Delta \mathrm{H}-\mathrm{b}-( \pm \Delta \mathrm{D})$ \\
Pago a empleados & $-(\mathrm{i}+\mathrm{l})-\mathrm{w}$ \\
Recaudo de intereses & $+\mathrm{d}-( \pm \Delta \mathrm{C})$ \\
Pago de intereses & $\pm \Delta \mathrm{I}-\mathrm{n}+\mathrm{w}$ \\
Pago de impuesto sobre las & $\pm \Delta \mathrm{K}-\mathrm{o}+\mathrm{t}$ \\
ganancias & $-\mathrm{y}-\mathrm{t}$ \\
Otros pagos & $+\mathrm{s}$ \\
Inversiones redimidas & $-\mathrm{t}$ \\
Nueva constitución de CDT & $+(\mathrm{f}-\mathrm{g}+\mathrm{e})$ \\
Recaudo por venta de equipo & $+\mathrm{u}$ \\
Nuevas obligaciones financieras & $-\mathrm{v}$ \\
Pago de obligaciones financieras & $-\mathrm{r}$ \\
Pago de dividendos & \\
\hline \hline & \\
Variación del efectivo y & $\pm \Delta \mathrm{A}$ \\
equivalentes al efectivo & \\
Tabla 23. Ecuación central 13 & \\
Fuente: elaboración propia &
\end{tabular}

\subsubsection{Fase 4. Presentación completa del EFE por el método directo}

Se toma la información contenida en la ecuación central 13, que contiene los flujos de efectivo propios del método directo, y se lleva a la presentación completa del EFE, lo cual se logra al hacer lo siguiente:

- En el extremo inferior, se cambia la expresión "Variación" por "Aumento", que corresponde 
a la situación del caso planteado de la empresa ADS; por lo cual le corresponde el signo positivo al monto de la variación del efectivo y equivalentes al efectivo; se opta por no colocarle signo, lo que indica que es positivo.

- Se adicionan los saldos inicial y final del efectivo y equivalentes al efectivo, tomados del ESF.

- Se anotan el título de cada actividad y sus respectivos subtotales.
- Se coloca el encabezado del estado financiero, indicando: nombre de la empresa, "Estado de Flujos de Efectivo" y el período que cubre, que para el caso planteado coincide con el del Estado de resultados.

Con lo anterior, se obtiene la presentación completa del EFE por el método directo, que se muestra en la tabla 24 .

\section{Empresa ADS}

Estado de Flujos de Efectivo

Desde el 1 de enero hasta el 31 de diciembre de 2015

Actividades de operación

Recaudo de clientes

Pago a proveedores

Pago a empleados

Recaudo de intereses

Pago de intereses

Pago de impuesto sobre las ganancias

Otros pagos

Flujo neto de efectivo de las actividades de operación

Actividades de inversión

Inversiones redimidas

Nueva constitución de CDT

Recaudo por venta de equipo

Flujo neto de efectivo de las actividades de inversión

Actividades de financiación

Nuevas obligaciones financieras

Pago de obligaciones financieras

Pago de dividendos

Flujo neto de efectivo de las actividades de financiación

Aumento del efectivo y equivalentes de efectivo

Saldo inicial del efectivo y equivalentes de efectivo

Saldo final del efectivo y equivalentes de efectivo
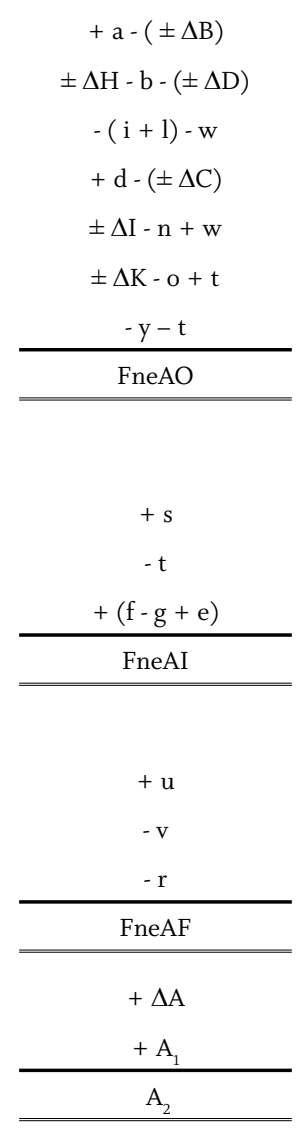

Tabla 24. Presentación completa del Estado de Flujos de Efectivo, EFE, por el método directo aplicado al caso planteado Fuente: elaboración propia 


\section{Conclusiones}

Con la utilización del lenguaje algebraico de los símbolos utilizados, manejando letras en lugar de los diferentes montos, se alcanza cierto nivel de generalización, con una gran gama de posibles resultados de las variaciones, ya sea con aumento o disminución; se logra detallar las situaciones planteadas en un caso de estudio, lo que fue necesario debido a que esto permite las explicaciones de un adecuado engranaje de las secuencias de las tareas y sus respectivos pasos. De no hacerlo y pretender una abstracción absoluta que abarcara la generalización total de la elaboración del EFE ajustable a todos los casos posibles, las explicaciones se harían demasiado extensas y confusas.

Las explicaciones del procedimiento para elaborar el EFE por el método directo, aplicado a un caso específico, constituyen una demostración matemática acompañada con argumentos contables y delimitados por la normativa contable contenida en la NIC 7.

El proceso de elaboración del EFE expuesto pasa por dos cambios trascendentales. El primero es el de la conformación de la ecuación preliminar de la variación del EFE, no solo porque se acerca a la estructura básica de presentación del EFE, sino porque el resultado de las variaciones como aumento (positivo) o disminución (negativo) deja de obedecer a la naturaleza de las cuentas donde se originan, y pasa a depender de su incidencia sobre el efectivo, ya sea a favor (positivo) o en contra (negativo) de este.

La segunda transformación valiosa obedece a la depuración de las variaciones, por la cual se configuran los flujos de efectivo y se elimi- nan las partidas que no los afectaron durante el período del EFE. Esto ocasiona la conversión de la información contable proveniente de la contabilidad de causación por acumulación (devengo) a aquella contabilidad de causación por caja.

Otro aspecto a destacar es la importancia de las políticas contables de cada empresa, que señalan el rumbo a seguir en la elaboración de los estados financieros, especialmente en aquellos aspectos en que la normativa contable da más de una alternativa en cuanto a la presentación y revelación. En su presentación, el EFE da la opción de ser presentado bajo el método directo o indirecto; además, permite que los flujos de efectivo que se derivan de los intereses y dividendos, y los pagos por impuesto a las ganancias, tengan alternativas para clasificarse en las actividades del EFE. Esto fue adecuadamente argumentado en las políticas contables del caso planteado, bajo un criterio coherente con respecto a su contabilización.

Las explicaciones expuestas bajo el lenguaje algebraico posibilitan su comprensión y aplicación a cualquier otro caso práctico del ámbito empresarial, para lo cual requiere el desarrollo de la habilidad de transferencia, considerada como una de las habilidades intelectuales de orden superior.

\section{Referencias}

Casallas-Gómez, Luz Marina \& Estrella, Claudia Patricia (2006). Actividades que potencian la interpretación de la letra como número generalizado: análisis de sus efectos en la clase de álgebra. En José V. Aymerich \& Sergio 
Macario Vives (eds.). Matemáticas para el siglo XXI, 125-132. Castellón de Plana, España: Universitat Jaume I.

Colombia, Ministerio de Comercio, Industria y Turismo, MinCIT (2012). Decreto 2784, por el cual se reglamenta la Ley 1314 de 2009 sobre el marco técnico normativo para los preparadores de información financiera que conforman el Grupo 1. Diario Oficial, 48.658, 29 de diciembre de 2012. Disponible en: http://www.mincit. gov.co/descargar.php?id=65489, http:// www.actualicese.com/normatividad/2012/ Decretos/Anexo-D2784-12.pdf

Colombia, Ministerio de Comercio, Industria y Turismo, MinCIT (2013). Decreto 3023, por el cual se modifica parcialmente el marco técnico normativo de información financiera para los preparadores de la información financiera que conforman el Grupo 1, contenido en el anexo del Decreto 2784 de 2012. Disponible en: http://www.mincit.gov.co/documentos. php?id=323, http://wsp.presidencia.gov. co/Normativa/Decretos/2013/Documents/ DICIEMBRE/27/DECRETO\%203023\%20 DEL\%2027\%20DE\%20DICIEMBRE\%20 DE\%202013.pdf

Consejo Técnico de Contaduría Pública, CTCP (2012). Direccionamiento estratégico del proceso de convergencia de las normas de contabilidad e información financiera y de aseguramiento de la información, con estándares internacionales. Disponible en: http://www. mincit.gov.co/descargar.php?id=66688

Díaz-Barriga Arceo, Frida \& Hernández-Rojas, Gerardo (2010). Estrategias docentes para un aprendizaje significativo. Una interpretación constructivista. México, D.F., México: McGraw-Hill.

Estupiñán-Gaitán, Rodrigo (2009). Estado de flujos de efectivo y de otros flujos de fondos. Bogotá: ECOE.

Frade-Rubio, Laura (2009). Desarrollo de las competencias lectoras y los obstáculos que se presentan. México: Inteligencia Educativa. Disponible en: http://docentesest121. wikispaces.com/file/view/Desarrollo+de+c ompetencias +lectoras $+\mathrm{y}+\mathrm{obst} \% \mathrm{C} 3 \% \mathrm{~A} 1 \mathrm{cul}$ os + que + se + presentan.pdf

García-Duque, Carlos Emilio (2006). Habilidades de pensamiento de orden superior, epistemología y evaluación en el aula de clase. Lúmina, 7, 8-26. Disponible en: http://www.umanizales.edu.co/ publicaciones/campos/economicas/lumina/ html/ediciones.html

García-Restrepo, Luis Enrique (2007). Lógica y pensamiento crítico. Manizales, Colombia: Universidad de Caldas.

Godino, Juan D. \& Font, Vicenç (2003). Razonamiento algebraico y su didáctica para maestros. Granada: Universidad Granada. Disponible en: http://www.ugr.es/ jgodino/ edumat-maestros/manual/7_Algebra.pdf Gómez-Marín, Raúl Antonio (2012). Breves tratados de lógica y argumentación: un enfoque integrado para humanistas. Medellín, Colombia: Fondo Editorial Universidad EAFIT. International Financial Reporting Standard, IFRS (2006). NIC 1 Norma Internacional de Contabilidad No. 1. Presentación de Estados Financieros. Disponible en: http:// www.nicniif.org/files/NIC\%201\%20 
Presentacion\%20de\%20Estados\%20

Financieros.pdf

International Financial Reporting Standard, IFRS (2006). NIC 7 Norma Internacional de Contabilidad No. 7. Estado de Flujos de Efectivo. Disponible en: http://www.nicniif. org/files/NIC\%207\%20Estados\%20de\%20 Flujo\%20de\%20Efectivo.pdf

McNamara, Daniel S. (2004). Aprender del texto: efectos de la estructura textual y las estrategias del lector. Revista Signos, 37 (55), 19-30. doi:10.4067/S0718. Disponible en: http://www.scielo.cl/scielo.php?pid=S071809342004005500002\&script=sci_arttext Muria-Vila, Irene Daniela \& Damián-Díaz, Milagros (marzo de 2008). Desarrollo de las habilidades de pensamiento en los diferentes niveles educativos. Revista Electrónica de Psicología Iztacala, 11 (1), 141151. Disponible en: http://www.iztacala. unam.mx/carreras/psicologia/psiclin/ vol11num1/vol11No1Art8.pdf

Ostengo, Héctor C. (2009). Cómo hacer un estado de flujo de efectivo a partir de los estados contables. Buenos Aires, Argentina: Librería Editorial Osmar D. Buyatti.

Pacter, Paul (enero de 2014). Global Accounting Standards - From Vision to Reality. Assessing the State of IFRS Adoption, Jurisdiction by Ju- risdiction. Disponible en: http://www.ifrs. org/Alerts/Publication/Documents/2014/ CPA-Journal-Global-Accounting-StandardsJanuary-2014.pdf

Petti, Ana María \& Longhi, Paula Ana (2007). Estado de flujo de efectivo. Buenos Aires, Argentina: Librería Editorial Osmar D. Buyatti.

Rincón-Soto, Carlos Augusto; Grajales-Londoño, Gherson \& Zamorano-Ho, Ricardo (2012). Estándares Internacionales en Contabilidad - EIC. Bogotá: Ediciones de la U. Sanz de Acedo-Lizarraga, María Luisa (2014). Competencias cognitivas en educación superior. Bogotá: Ediciones de la U.

- Fecha de recepción: 14 de marzo de 2014

- Fecha de aceptación: 17 de abril de 2015

- Disponible en línea: 30 de abril de 2015

\section{Para citar este artículo}

Duque-Sánchez, Alicia Patricia (2015). Estado de flujos de efectivo: aplicación de razonamientos algebraicos y de la NIC 7. Cuadernos de Contabilidad, 16 (40), 73 110. http://dx.doi.org/10.11144/Javeriana. cc16-40.efea 